\title{
LARGE-SCALE SIMULATIONS OF PHASE TRANSITIONS AND LOW-DIMENSIONAL MAGNETS
}

\author{
G. KA M I E N I A R Z \\ Institute of Physics, Adam Mickiewicz University \\ ul. Umultowska 85, 61-614 Poznań, Poland \\ e-mail: gjk@pearl.amu.edu.pl
}

\begin{abstract}
Recent developments in computer simulations of phase transitions in Ising-like systems and thermodynamic behaviour of quantum spin chains are reviewed. A combination of stochastic Monte Carlo as well as deterministic transfer matrix and finite-size diagonalization methods is described in both fields as regards static properties. Asymptotic analysis and extrapolation techniques are presented in detail. Some effective-field methods, series expansions, spin dynamics simulations and experimental applications are also discussed.
\end{abstract}

\section{Introduction}

Phase transitions and critical phenomena have been the subject of intense study for a few decades. It has led to the concept of universality, scaling invariance and the idea of a small number of critical exponents. Only a limited number of exact solutions has been found so that main results are based on approximate solutions (such as series expansions and renormalisation group theory) and numerical techniques.

Recent progress in computer technology and development of new algorithms for Monte Carlo simulations together with new methods (Landau, 1994) of analysis have yielded results of high resolutions competing with the series expansion and renormalisation group data.

The role of computer simulations in the field of low-dimensional quantum spin systems has also increased since the pioneering work of Bonner and Fisher (1964). Particulary interesting are the applications to some soliton-bearing and Haldane-gap systems.

For a classical system one can describe the states of the system and their energies in terms of a single set of variables, like the $z$-components of the spin in the Ising model. Each configuration of the variables appears with the Boltzmann 
probability and the problem is to sample the configurations according to the Boltzmann distribution.

Generalization of the Monte Carlo technique to quantum machanical problem is not an easy task. In this case the states and energies are the eigenstates and eigenvalues of Hamiltonian operator acting on the Hilbert space. In many cases it is even not possible to describe exactly these eigenstates.

This article is not intended to be exhaustive. It is rather aimed at reviewing simulation studies the author has been involved in. The report is organized as follows. In Section 2 some simulations methods are described and in Section 3 the numerical analysis of simulations is discussed. Selected results for some classical and quantum systems are presented in Section 4. The report is closed with some conclusions.

\section{Methods of simulations}

\subsection{Classical Monte Carlo}

This method is applied here to the three dimensional ferromagnetic Ising model with the Hamiltonian

$$
H=-J \sum_{<i, j>} \sigma_{i} \sigma_{j}
$$

where the spin $\sigma_{i}= \pm 1$, the sum runs over all nearest-neighbour pairs and the periodic boundary conditions are imposed.

Traditionally, Monte Carlo simulations were permformed using the single spin-flip method of Metropolis which becomes rather inefficient near phase transitions due to critical slowing down. To improve the performance, the multispin coding method was devised but it did not eliminate critical slowing down (Landau, 1994). Only recently a number of cluster-flipping methods accomplished its significant reduction. Swendsen and Wang (1987) found an algorithm based on a theorem mapping the Potts model into a percolation model. At first an initial spin system is split into a set of correlated clusters and then it is flipped randomly with probability $1 / 2$. Further improvement is due to Wolff (Wolff, 1989). According to this approach, a single cluster is growing at first and then it is flipped. In our simulations (Blöte and Kamieniarz, 1993) the cluster methods are applied.

\subsection{Transfer matrix}

The transfer matrix method is a powerful tool of simulations. In the case of an Ising-like model located on a square lattice $L_{X} \times L_{Y}$ with periodic boundaries and nearest-neighbour interactions the tranfer matrix is introduced as follows 
(Kamieniarz and Blöte, 1993a). The spins belonging to the $j$ th column are denoted by $\vec{S}_{j}=\left(S_{j} 1, S j_{2}, \ldots, S_{j L_{y}}\right)$ so that

$$
Z=\sum_{\vec{S}_{1}, \cdots, \vec{S}_{L_{x}}} \exp \left[-\beta \mathcal{H}\left(\vec{S}_{1}, \cdots, \vec{S}_{L_{x}}\right)\right]=\operatorname{Tr} \mathbf{T}^{L_{x}}
$$

where the transfer matrix $\mathrm{T}$ is defined in the $2^{l \mathrm{y}}$ - dim manifold of all configurations of $L_{y}$ spins

$$
S_{j k}= \pm 1, k=1, \cdots, L_{y}
$$

The transfer matrix $\mathbf{T}$ can be split into a product

$$
\mathbf{T}=\mathbf{T}_{v} \mathbf{T}_{h}
$$

where

$$
\mathbf{T}_{v}\left(\vec{S}_{i}, \vec{S}_{j}\right)=\exp \left[\sum_{k=1}^{L_{y}}\left(K_{y} S_{j k} S_{j, k+1}+h S_{i k}\right)\right] \delta_{\vec{S}_{i}, \vec{S}_{j}}
$$

is diagonal, whereas

$$
\mathbf{T}_{h}\left(\vec{S}_{i}, \vec{S}_{j}\right)=\exp \left(K_{x} \sum_{k=1}^{L_{y}} S_{i k} S_{j k}\right)
$$

is non-diagonal and can be expressed as a product of sparse matrices.

The tranfer matrix enables not only evaluation of the partition function but also the magnetization moments which enter the Binder cumulant (Binder, 1981). From the expansion

$$
Z(h)=\sum_{k=0}^{\infty} \frac{h^{k}}{k !} Z_{k}
$$

where

$$
Z_{k}=\left(\frac{\partial^{k} Z}{\partial h^{k}}\right)_{h=0}
$$

the corresponding moments of the magnetization can be expressed as

$$
\left\langle M^{k}\right\rangle_{h=0}=Z_{k} / Z_{0}
$$

To calculate accurately the lower order moments (8), it suffices to find the corresponding coefficients $\mathrm{Zk}$ within a perturbative scheme, i.e. to expand the diagonal matrix $\mathrm{T}$, in terms of $h$ and to keep track of the powers of $h$ during successive multiplications of a given vector by $\mathbf{T}$. 


\subsection{Quantum Monte Carlo and transfer matrix}

The finite-temperature quantum Monte Carlo $(Q M C)$ method has been applied (Suzuki, 1993) to a number of 1-dim and 2-dim spin systems. Here we consider 1-dimensional chains described by the anisotropic Heisenberg Hamiltonian with nearest - neighbour interactions (Cullen and Landau, 1983; Kamieniarz, Mallezie and Dekeyser, 1988)

The starting point of $Q M C$ method is the generalized Trotter formula for the exponential of a sum of $k$ noncommuting operators

$$
\exp \left(\sum_{i=1}^{k} \hat{\theta}_{i}\right)=\lim _{m \rightarrow \infty}\left[\prod_{i=1}^{k} \exp \left(\frac{\hat{\theta}_{i}}{m}\right)\right]^{m},
$$

where $m$ is an integer referred to as the "Trotter index".

One can apply this formula to the partition function and map a given $1 d$ quantum model into a classical $2 d$ system which can be studied using classical Monte Carlo or transfer matrix methods. The mapping is accomplished in the following way. The partition function $Z$ is expressed in terms of all the eigenstates of the spin chain and the Hamiltonian is partitioned into a sum of two-body operators

$$
H=\sum_{n=1}^{N} H_{n, n+1}=H_{o d d}+H_{e v e n}
$$

where

$$
H_{\text {odd }(e v e n)}=\sum_{n=o d d(e v e n)} H_{n, n+1}
$$

In the checker-board decomposition ( $C B D$ ) (Kamieniarz et al., 1988) the Hamiltonian is split into two parts $\hat{H}_{\text {odd }}$ and $\hat{H}_{\text {even }}$, containing the two-body operators with odd and even $n$, respectively. The mth-order approximant to the partition function is then given by

$$
Z_{C B D}^{(m)}=\operatorname{Tr} \cdot\left[\left(\hat{L}_{\text {odd }} \hat{L}_{\text {even }}\right)^{m}\right]
$$

where

$$
\hat{L}_{o d d(\text { even })}=\prod_{n=o d d(e v e n)} \exp \left(-\frac{\beta}{m} H_{n, n+1}\right)
$$

By introducing the complete sets of states

$$
\left|\sigma_{r}>=\right| S_{1 r}, S_{2 r}, \ldots, S_{N r}>(r=1,2, \ldots, 2 m)
$$

one gets

$$
Z_{C B D}^{(m)}=\sum_{\sigma_{1}, \ldots, \sigma_{2 m}}<\sigma_{1}\left|\hat{L}_{o d d}\right| \sigma_{2}><\sigma_{2}\left|\hat{L}_{e v e n}\right| \sigma_{3}>\ldots \ldots<\sigma_{2 m}\left|\hat{L}_{e v e n}\right| \sigma_{1}>
$$


where

$$
\begin{gathered}
<\sigma_{r}\left|\hat{L}_{\text {odd(even) }}\right| \sigma_{r+1}>= \\
\prod_{n=\text { odd (even) }}<S_{n T} S_{n+1, r}\left|\exp \left(-\frac{\beta}{m} H_{n, n+1}\right)\right| S_{n, r+1} S_{n+1, r+1}>=\prod_{n=o d d(\text { even })} e^{-\beta E_{n, r}}
\end{gathered}
$$

and $r$ is odd (even) for $\hat{L}_{\text {odd }}\left(\hat{L}_{\text {even }}\right)$. This leads to the expression for a classical partition function

$$
Z_{C B D}^{(m)}=\sum_{\sigma_{1}, \ldots \sigma_{2 m}} \prod_{\langle n, r>} e^{-\beta E_{n, r}}
$$

for a $2 d$ lattice of size $N \times 2 m$, where $(n, r)$ indicates that $n$ and $r$ are both odd or both even. Because of the trace in $Z$, we have periodic boundary conditions in the Trotter direction. Imposing the periodic boundary conditions on the chain direction, the $2 d$ lattice consists of $N$ x $m$ blocks of four spins ("vertices") (Kamieniarz et al., 1988) and the quantity

$$
W_{n r}=e^{-\beta E_{n, r}}
$$

is called the weight of the vertex.

In the real-space decomposition ( $R S D$ ) (Kamieniarz et al., 1988) one applies the Trotter formula to the partition function using the representation $\hat{H}=\sum_{n=1}^{N} H_{n, n+1}$. Again a partition function of a classical spin - $1 / 2$ Ising system on a $2 d$ lattice with $N$ x $m$ vertices is obtained.

\subsection{Finite-size diagonalization}

Exact calculations on small finite-size lattices are considered an important complement to and a test of computer simulations on larger lattice systems (Manousakis, 1991). Although limited to substantially smaller sizes, they do not encounter such possible sources of errors in simulation studies as the Trotter approximation or metastability in Monte Carlo sampling.

The calculations proceed as follows (Bonner et al., 1964; Blöte, 1975). The matrix representation of a given spin Hamiltonian is found and diagonalized numerically for finite chains with size $N$ up to $N_{\max }$ and free boundary conditions. For a given size, interesting thermodynamic functions are then evaluated according to statistical mechanics.

\subsection{Spin dynamics}

An interest in dynamics of the ferromagnetic easy-plane Heisenberg chains stems from spin solitons (Mikeska, 1978) predicted theoretically for the $I d$ model in 
the symmetry breaking field which can be mapped under certain approximations onto the classical sine-Gordon model, yielding nonlinear excitations. In a reliable computer experiment with all microscopic parameters controlled and all polarizations directly measured, new insight into the validlity of the sine-Gordon model can be expected (Gerling and Landau, 1990).

The dynamics of models with continuous degrees of freedom can be derived directly from equations of motion (Grille, Kamieniarz and Gerling, 1992). In the first stage a standard importance sampling Monte Carlo technique is used to generate equilibrium spin configurations for particular values of $T$ and $B$ with chain length of typically $N=20000$ sites and periodic boundaries. Next the coupled nonlinear equations of motion for the spin variables are accurately integrated up to time $t_{m_{a x}}=100 / \mathrm{J}$ with a time integration interval $\Delta=0.01 / \mathrm{J}$. For each time integration, the time and space dependent spin-spin correlation functions

$$
<S_{i}^{\alpha}(0) S_{i+r}^{\alpha}(t)>=\frac{1}{N} \sum_{i=1}^{N} S_{i}^{\alpha}(0) S_{i+r}^{\alpha}(t)-<S_{i}^{\alpha}(0)>^{2}
$$

are calculated and averaged over the 10 starting configurations. Finally, the time and spatial Fourier transforms are evaluated to yield the dynamic structure factors $S(q, w)$, where $(=x, y, z)$ denotes polarization. The same technique is also reviewed by Landau (1994) in relation to the critical dynamics simulations.

\section{Methods of analysis}

\subsection{Finite-size scaling}

We consider a system with finite-size parameter $L$, e.g. a hypercube of size $L^{d}$ with toroidal boundary conditions. In the vicinity of a renormalisation fixed point, the parameters describing the system include the temperature field $t$, the magnetic field $h$ and the finite-size field $1 / \mathrm{L}$. Neglecting the irrelevant fields and nonlinearities, the asymptotic finite-size scaling relation for the singular part of the free energy per spin (Privman and Fisher, 1984; Barber, 1983) can be written as

$$
f^{(s)}(t, h, 1 / L)=b^{-d} f^{(s)}\left(t b^{y_{t}}, h b^{y_{h}}, b / L\right)
$$

where $b$ is the rescaling factor, and $y_{t}$ and $y h$ are the bulk thermal and magnetic exponents, respectively. This finite-size scaling relation has been widely applied in numerical studies of critical phenomena and in analysing experimental data. Choosing $b=L$, differentiating $k$ times with respect to $h$ and putting $h=0$,

$$
f^{(s), k}(t, 0,1 / L)=L^{k y_{h}-d} f^{(s), k}\left(t L^{y_{t}}, 0,1\right)
$$


The scaling function on the right hand side represents a system far from criticality (the finite-size parameters has the value 1) and is therefore assumed analytic, and may be Taylor expanded in $t L^{y t}$. Expressing the magnetization moments $\left(\mathrm{M}^{k}\right)$ in derivatives of the free energy, neglecting the analytic part, one shows that these moments obey the same scaling behaviour as above. Thus, in the vicinity of the fixed point ( $L$ large, $t$ small) the ratio

$$
Q_{L} \equiv\left\langle M^{2}\right\rangle_{L}^{2} /\left\langle M^{4}\right\rangle_{L}
$$

satisfies

$$
Q_{L}(t)=Q+a_{1} t L^{y_{T}}+a_{2} t^{2} L^{2 y_{T}}+\ldots
$$

where $Q$ and $\mathrm{a}_{\mathrm{i}}$ are unknown parameters.

Another unknown is the critical temperature $T_{c}$ which enters via $t \sim T-T_{c}$. The unknowns can be determined by fitting (23) to the Monte Carlo data. If $Q$ is known, one unknown parameter is eliminated, so that the critical point can be obtained more accurately.

The finite-size scaling relation has to be modified (Kamieniarz et al., 1993a) for the $2 d$ Ising model which is particular in some respects. Firstly, the specific heat singularity has a logarithmic divergence so that the zero-field free energy should contain explicitely a logarithmic term (Niemeijer and van Leeuwen, 1976). Secondly, it has been argued (Aharony and Fisher, 1983) that the leading corrections to scaling are analytic and can be accounted for by nonlinearities of the scaling fields $g_{t}$ and $g_{h}$ related to the thermal field $t$ and the ordering field $h$. It can be arranged that the renormalisation equations which are nonlinear in $t$ and $h$, become linear in variables $g_{t}$ and $g_{h}$. Thus under rescaling by a factor of $b$

$$
g_{t}^{\prime}=b^{y_{t}} g_{t}, \quad g_{h}^{\prime}=b^{y_{h}} g_{h}
$$

where the primes denote the renormalised quantities. Furthermore, we have for the finite-size field

$$
1 / L \rightarrow 1 / L^{\prime}=b / L,
$$

so that for the $2 d$ Ising model, which has $y_{t}=1$, the fields $g_{t}$ and $1 / L$ fulfil the same relation. Their ratio $g_{t} L$ is invariant under rescaling and, along a trajectory with constant $\mathrm{g}_{t} L$ one may combine $\mathrm{g}_{t}$ and $1 / L$ into a single field proportional to $1 / L$, keeping in mind that the critical amplitudes may still depend on $g_{t} L$.

Along this trajectory the known results (Aharony et al., 1983) for the scaling behaviour of the free energy in terms of $\mathrm{g}_{t}$ and $\mathrm{g}_{h}$ can be generalized and the corresponding singular part $F^{(\mathrm{s})}$ of the total free energy is thus expressed as

$$
F^{(s)}\left(g_{t}, g_{h}, L^{-1}\right)=A\left(g_{t} L\right) \ln L+B\left(g_{t} L, g_{h} L^{y_{h}}\right)
$$


where $A$ and $B$ are unknown amplitudes and the nonlinear fields are expanded as

$$
\begin{gathered}
g_{t}=t+b_{t} h^{2}+c_{t} t^{2}+\cdots \\
g_{h}=h\left(1+c_{h} t+d_{h} t^{2}+e_{h} h^{2}+\cdots\right)
\end{gathered}
$$

The scaling form of the free energy enables the calculation of derivatives with respect to the field at the critical point $t=0, h=0$. From the relations

$$
\left\langle M^{2}\right\rangle=\frac{\partial^{2} F}{\partial h^{2}}, \quad\left\langle M^{4}\right\rangle=\left[\frac{\partial^{4} F}{\partial h^{4}}+3\left(\frac{\partial^{2} F}{\partial h^{2}}\right)^{2}\right]
$$

we obtain (Kamieniarz et al., 1993a) the following expansion for $Q_{L}$ up to $L^{3-4 y h}$ :

$$
\begin{gathered}
Q_{L}=Q+\beta_{0} L^{2-2 y_{h}}\left(1+\beta_{1} L^{-1}+\beta_{2} L^{2-2 y_{h}}+\beta_{3} L^{-2}+\right. \\
\left.\beta_{4} L^{1-2 y_{h}}+\cdots\right)+\gamma_{0} L^{1-2 y_{h}} \ln L\left(1+\gamma_{1} L^{2-2 y_{h}}+\cdots\right)
\end{gathered}
$$

This expansion contains not only a number of algebraic powers, but also logarithmic terms which may complicate the determination of $Q$ from the finitesize results.

\subsection{Conformal invariance}

Conformal invariance can be exploited to relate the properties of spin models on strips of finite width $L$ and infinite length with periodic boundary conditions to those of the corresponding $2 d$ system. A relation derived by Cardy (1987) applies to the finite-size amplitude of the correlation length

$$
\lim _{L \rightarrow \infty} \frac{L}{\xi_{i}}=2 \pi x_{i}
$$

where $x_{i}=d-y_{i}$ is the anomalous dimension of the observable associated with the correlation length The scaled gaps

$$
L g_{i}(L) / 2 \pi
$$

where

$$
g_{i}(L)=\xi_{i}^{-1}(L)=\ln \frac{\lambda_{0}}{\left|\lambda_{i}\right|}
$$

provide estimates of $x_{i}$ which can be evaluated from power-law extrapolations. 


\subsection{Finite-size extrapolations}

Estimates of thermodynamic quantities for macroscopic systems can be found from the finite-size data $\mathbf{A j v}$ by recourse to extrapolation procedures (Bonner et al., 1964; Blöte, 1975; de Neef, 1976). The series $A N$ have been mainly analysed in terms of polynomial expansions. These methods assume (Campana, Caramico D'Auria, Esposito, Kamieniarz and Dekeyser, 1990) that the An may be approximated by expressions of the form

$$
A_{N} \simeq A_{N}(n)=\sum_{k=0}^{n} B_{k} y_{N}^{k}
$$

where are obtained by minimizing the mean square deviation

$$
\Delta^{2}=\sum_{N=N_{1}}^{N_{2}}\left[A_{N}-A_{N}(n)\right]^{2} / M, \quad M=N_{2}-N_{1}+1
$$

and $y_{N}=f(1 / \mathrm{N})$, with a function $f(x)$ fulfilling the property $f(0)=0$. The parametr $B_{0}$ is clearly the estimate for $A$ in these methods. Apart from the magnitude of ${ }^{2}$, the quality of this analysis can be estimated from the rate of convergence obtained for $B_{k}$ when the lowest index $N_{1}$ is changed.

The polynominal extrapolations usually performed, correspond to the choice $y_{N}=1 / \mathrm{N}$. Because of the limited number of $A_{N}$ values, the results may not be improved by increasing the order $n$ of the polynominal as the higher-order coefficients $\mathrm{B}_{k}$ do not always remain small. In practice, the series have been analysed by linear $(n=1)$ and quadratic $(n-2)$ fits.

Obvious choices are also simple powers $f(x)=x^{\alpha}(\alpha>0)$, or $f(x)=1 / \ln (x)$. One tries to obtain a better convergence by first transforming the variable $1 / N$ into $y_{N}=f(1 / N)$ and then by fitting the $A n$ data to a low-order polynominal of the form (32). In our analysis (Campana et al., 1990) however, the best results in terms of acceptable estimates with low ${ }^{2}$ values were obtained with the form

$$
f(x)=\left(e^{\alpha x}-1\right) / \alpha,
$$

or $f(x)=x /(1-\alpha x)$. In each case, is a free parameter again determined by minimizing $\Delta^{2}$. Although formally equivalent to the previous method (expansions in powers of $1 / N$ ), it has the advantage of allowing the incorporation of higher-order terms in a low-order expansion.

Some relations have been rigorously established for the finite-size estimates. The asymptotic form of the specific heat per site $C_{N}$ calculated for the chains of size $N$ depends on the boundary conditions (de Neef, 1976). For free boundary conditions:

$$
C_{N}^{c h a i n}=C_{\infty}-\frac{b}{N}+0\left(\beta^{2 N+2}\right)
$$


where the correction term has an alternating effect depending on the even or odd value of $\mathrm{N}$, whereas for periodic boundary conditions:

$$
C_{N}^{\text {ring }}=C_{\infty}+\frac{\Phi_{N}}{N}+0\left(\beta^{N}\right)
$$

where not only the correction term is of the lower order with respect to $N$ but also the second term depends on N. For antiferromagnetic interactions $\Phi_{N}$ is alternating in sign for even and odd $\mathrm{N}$, whereas for ferromagnetic exchange - it is uniform in sign.

As to the QTM finite-m results, (Delica and Leschke, 1990) the behaviour

$$
C_{m}=C_{\infty}+\sum_{n=1} \frac{a_{n}}{m^{2 n}}
$$

is expected.

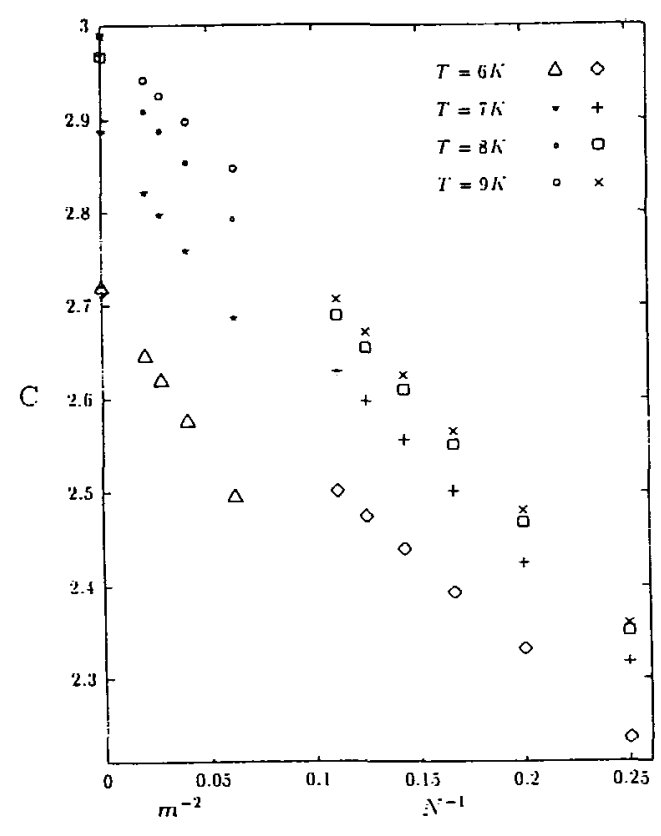

Figure 1: Variation of the zero-field specific heat $C$ in molar units $(J /$ mole $K$ ) against $1 / m^{2}$ (the transfer matrix data, $4 \leq m \leq 7$ ) and $1 / N$ (the finite-chain diagonalizatioin data, $4 \leq N \leq 9$ ). The symbols on the ordinate axis shows the corresponding extrapolated values.

The $1 / \mathrm{N}$ and $1 / \mathrm{m}^{2}$ dependence of the thermodynamic quantities in the low temperature region for the ferromagnetic $S=1$ easy-plane Heisenberg chain is illustrated in Figs. 1-3 for the microscopic parameters $J / \mathrm{k}_{B}=20.5 \mathrm{~K}$ and $A / J=0.425$ found by Campana, Caramico D'Auria, Esposito, Esposito, Gerling 
and Kamieniarz (1992a). The variation of the molar finite-size specific heat (Campana, Caramico D'Auria, Esposito, Esposito and Kamieniarz, 1996) with respect to $1 / N$ is plotted for temperatures $6 \leq T \leq 9 K$ in Fig. 1 . In the entire temperature range the $1 / N$ dependence shows linear behaviour so that the extrapolated data can be extracted with the accuracy of an order of $0.1 \%$ and within this error they agree with the transfer matrix data plotted versus $1 / m^{2}$ in the same figure.

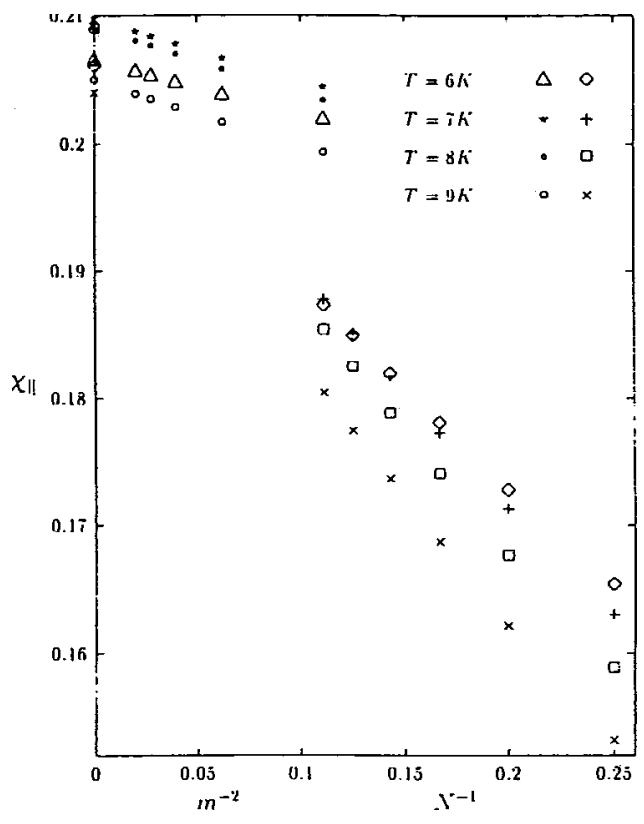

Figure 2: Variation of the longitudinal susceptibility $\chi_{\|}$in the molar units (emu/mole) against $1 / m^{2}$ (for the transfer matrix data, $3 \leq m \leq 7$ ) and $1 / N$ (for the finite-chain diagonalization data, $4 \leq N \leq 9$ ). The extrapolated values are depicted on the ordinate axis.

The relation (35) refers only to the specific heat, nevertheless we also analyse (Campana et al., 1996) other thermodynamic quantities in terms of the inverse length $N^{-1}$. As can be seen from Fig. 2, even in our low temperature region $(T \leq 9 K)$ the linear dependence of $\chi_{\|}$for the finite-chain data in $1 / N$ and that of the QTM results in $1 / \mathrm{m}^{2}$ is well fulfilled. As to the excess specific heat $\Delta C=C(T, B)-C(T, B=0)$, the corresponding size dependence is illustrated in Fig. 3.

Qualitatively the same finite-size behaviour is observed for systems with antiferromagnetic interactions. In Fig. 4 a typical size dependence of susceptibility is shown for a model of the Haldane-gap compound $\mathrm{CsNiCl}_{3}$ (Kamieniarz and 


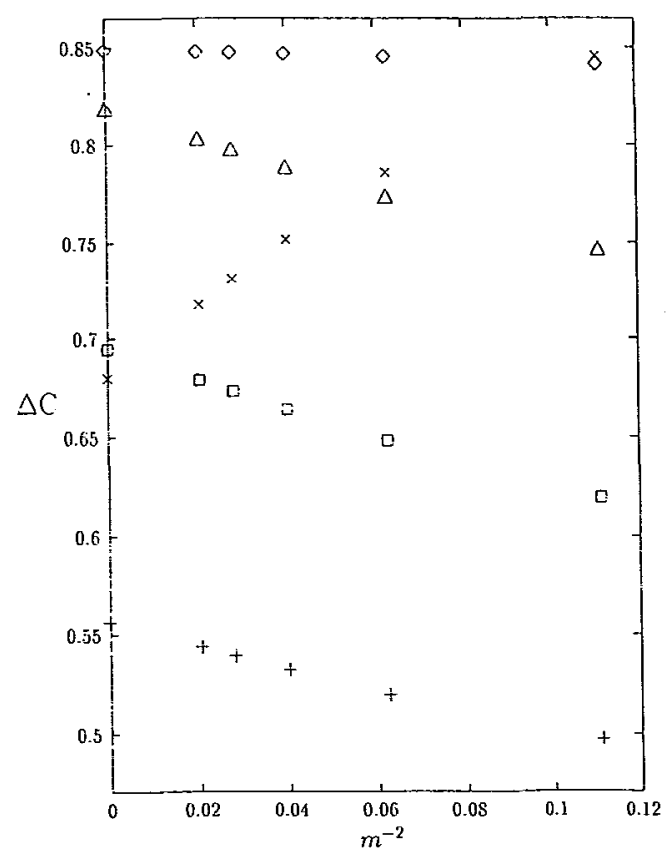

Figure 3: Dependence on $1 / \mathrm{m}^{2}$ of the excess specific heat $\Delta C(\mathrm{~J} / \mathrm{mole} K)$ in the field $B=$ $0.5 k$ Gs. The symbols $\times, \diamond, \triangle, \square,+$ display the data at $T=6,7,8,9,10 K$, respectively. The extrapolated predictions are illustrated on the ordinate axis.

Matysiak, 1996a).

The finite-size data can also be analysed (Campana et al., 1990) in the framework of the Pade approximant method. This method is based on the fact that the limit $A_{\infty}$ of a series $A_{N}$ can be obtained from

$$
A_{\infty}=\left.(1-z) F(z)\right|_{z=1}
$$

where

$$
F(z)=\sum_{N=0}^{\infty} A_{N} z^{N}
$$

so that

$$
(1-z) F(z)=A_{0}+\sum_{N=1}^{\infty}\left(A_{N}-A_{N-1}\right) z^{N}
$$

From the coefficients $A_{N}$ one can build Pade approximants to $(1-z) F(z)$ and get estimates of $A_{\infty}$. A variant of the method is the alternating $\varepsilon$-algorithm described by Barber (1983). 


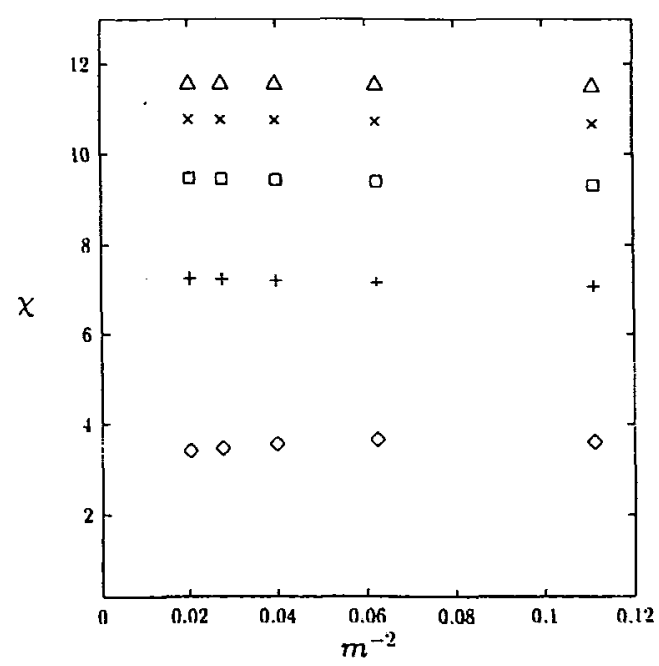

Figure 4: Variation of $\chi_{\perp}(e m u / m o l e)$ for negative $\mathrm{J}$ against $1 / m^{2}(3 \leq m \leq 7)$ and $1 / N$ $(4 \leq N \leq 8)$. The corresponding extrapolated values are given on the ordinate axis.

Another procedure is to construct new series (Bonner et al., 1964; Blöte, 1975). If the series $A_{N}$ consists of a number of power-law terms $N^{X i}(i=$ $1,2, \ldots$ ), a given power $N \sim^{x}$ is eliminated (Kamieniarz et al., 1993a) in the new series defined as

$$
A_{N}^{\prime}=\left[(N+1)^{x} A_{N+1}-N^{x} A_{N}\right] /\left[(N+1)^{x}-N^{x}\right]
$$

In the modified series $A_{N}^{\prime}$ the power-law behaviour is preserved with the same constant $A_{\infty}$, but without the term containing $N^{-x}$.

\subsection{Effective fields with correlations}

A possible generalization of the mean-field approximation for a system with short-range interactions can be introduced (Dekeyser and Kamieniarz, 1992) as follows. We divide the lattice into a finite cluster $\Omega$, its boundary $\partial \Omega$ and the complement $\bar{\Omega}$, as shown in Fig. 5. The corresponding spin degrees of freedom are denoted by the variables $\sigma \in \Omega, \tau \in \partial \Omega, \bar{\sigma} \in \bar{\Omega}$. The Hamiltonian of a system is split into the form

$$
-\beta \mathcal{H}(\sigma, \tau, \bar{\sigma})=H_{0}(\sigma, \tau)+H_{1}(\tau, \bar{\sigma})
$$




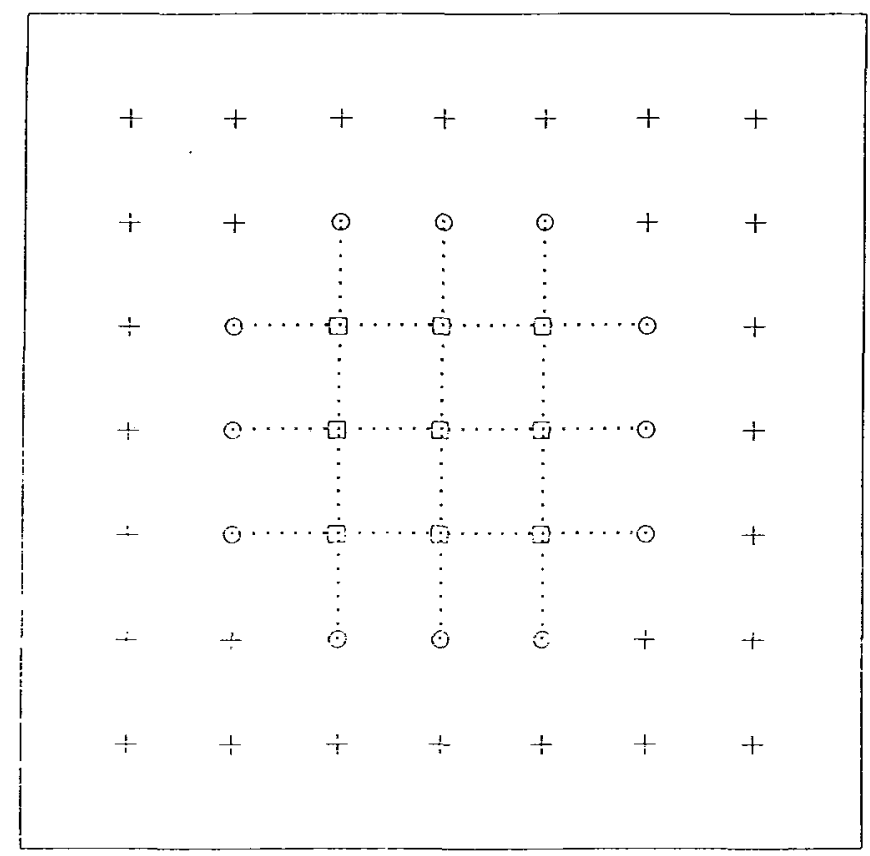

Figure 5: Partition of the lattice into the cluster $\Omega$, the boundary $2 \Omega$, and the complement $\bar{\Omega}$.

and we address a problem of estimating the thermal expectation value

$$
<A>=\frac{1}{Z} \operatorname{Tr} e^{-\beta H(\sigma, \tau, \bar{\sigma})}
$$

For a classical system the average

$$
<A>=\frac{\sum_{\sigma \tau} A(\sigma, \tau) \mathrm{e}^{H_{0}+H^{\prime}(\tau)}}{\sum_{\sigma \tau} \mathrm{e}^{H_{0}+H^{\prime}(\tau)}}=\frac{\sum_{\sigma \tau} A \mathrm{e}^{H_{0}} Q(\tau)}{\sum_{\sigma \tau} \mathrm{e}^{H_{0}} Q(\tau)}
$$

can be expressed in terms of the effective Hamiltonian $H^{\prime}(\tau)$, where

$$
\mathrm{e}^{H^{\prime}(\tau)}=\sum_{\bar{\sigma}} \mathrm{e}^{H_{1}(\tau, \bar{\sigma})} \equiv Q(\tau)
$$

The simplest approximation of , is the mean-field approximation, i.e. a dependence linear in $r$ variables. However, higher order terms in $r$ can be also imposed according to the symmetry of $\mathcal{H}$. These new terms contain correlations in $T$ variables.

The function $Q(\tau)$ can be expanded in a form (Dekeyser et al., 1992)

$$
Q(\tau)=\prod_{i}\left(1+a_{i} \tau_{i}\right) \prod_{i \neq j}\left(1+b_{i j} \tau_{i} \tau_{j}\right) \ldots
$$


where the coefficients $b_{i j}$ take into account the correlations between the spins of $\partial \Omega$. The symmetry properties of the cluster are reflected in the symmetry properties between these coefficients.

Then a sequence of systematically improvable approximations can be worked out (Kamieniarz, Musial and Dekeyser, 1994), imposing selfconsistent conditions on the coefficients in eq.(46) for $Q$ (in analogy with the usual mean field approximation) or implementing the renormalisation-group ideas (in analogy with the mean-field renormalisation group method (Indekeu, Maritan and Stella, 1982) denoted as MFRG) which lead to non-classical critical exponents.

The critical couplings $K_{c}=J / k_{B} T_{c}$ for the Ising clusters with $L^{2}$ sites on the square lattice are given in Table 1 . In the second and the third column, the cluster mean-field approximation results and the original MFRG predictions (Indekeu et al., 1982) are given. Performing the selfconsistent calculations (Kamieniarz, Musial and Dekeyser, 1996b) with two parameters in $Q(\mathrm{r})$ for the pair correlations of r, we find the results for $K_{C}$ given in the 4 th and 5 th columns of Table 1 for the $Q$ and the renormalisation-group scheme, respectively. A much faster convergence is obtained by increasing the order of the approximation than by increasing the cluster size in MFA. The corresponding critical exponents $y_{t}$ and $\mathrm{y}_{\mathrm{h}}$ for the thermal and ordering fields are given in the remaining part of Table 1. The values are considerably improved, in particular the exponents $y_{t}$.

Table 1: The critical coupling $K_{c}$ and the critical exponents for the $2 d-$-Ising model.

\begin{tabular}{|c|c|c|c|c|c|c|c|c|}
\hline \multirow[t]{2}{*}{$\mathrm{L}$} & MFA & MFR & $Q^{s c}$ & Qrg & MFR & $Q^{T 9}$ & MFR & Qrg \\
\hline & \multicolumn{4}{|c|}{$K_{c}$} & \multicolumn{2}{|c|}{$y_{t}$} & \multicolumn{2}{|c|}{$\mathrm{y}_{\mathrm{h}}$} \\
\hline 1 & 0.250 & & & & & & & \\
\hline 2 & 0.286 & 0.361 & 0.412 & & 0.69 & & 1.50 & \\
\hline 3 & 0.308 & 0.381 & 0.414 & 0.409 & 0.78 & 0.972 & 1.57 & 1.69 \\
\hline 4 & 0.323 & 0.393 & 0.417 & 0.412 & 0.82 & 0.989 & 1.60 & 1.69 \\
\hline 5 & 0.335 & 0.401 & & & 0.84 & & 1.62 & \\
\hline
\end{tabular}

The qualitatively different situation appears for quantum mechanical models. The Boltzmann factor cannot be factorized so that an effective Hamiltonian $H^{\prime}(\sigma, \tau)$

$$
\mathrm{e}^{H_{0}+H^{\prime}(\sigma, \tau)}=\sum_{\bar{\sigma}} \mathrm{e}^{H_{0}+H_{1}(\tau, \bar{\sigma})}
$$

may also depend on the variables $\sigma$ from the interior of the cluster $\Omega$.

We assume (Dekeyser et al., 1992) that the strongest contributions to $H^{\prime}(\sigma, \tau)$ appear at or around the boundary $\partial \Omega$ and we choose them according to the symmetry of $\mathcal{H}$. 
For simple $1 d$ Heisenberg Hamiltonian without external field, no broken symmetry can be expected and we can argue that for symmetry reasons no external field can appear in $H^{\prime}$. The first contribution that can be expected in $H^{\prime}$ is an extra Heisenberg interaction between the first and the second spin in a finite segment, and equally between the last and the last but one:

$$
H_{0}+H^{\prime}=-\frac{1}{2} \sum_{i=0}^{N-1} K_{i}\left(\sigma_{i}^{x} \sigma_{i+1}^{x}+\sigma_{i}^{y} \sigma_{i+1}^{y}+\sigma_{i}^{z} \sigma_{i+1}^{z}\right),
$$

where $K_{0}=K_{N}=K+D$ and $K_{i}=K$ for all other $i$.

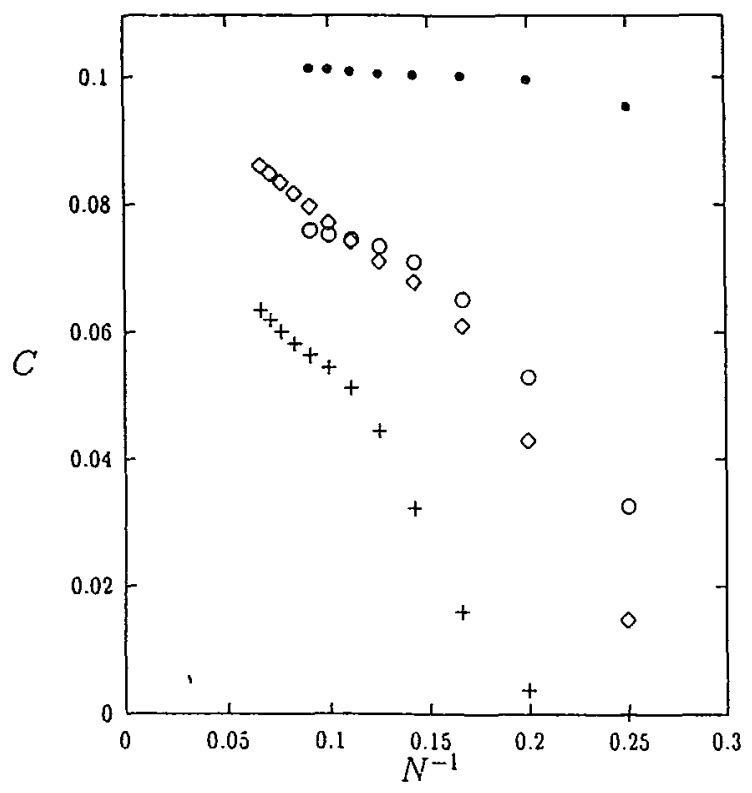

Figure 6: The finite-size results for the zero-field specific heat of the $1 d$ Heisenberg model. The symbols $\bullet \diamond$ represent the standard and improved data at $K^{-1}=0.1$, whereas o, +- the corresponding data at $K^{-1}=0.05$.

Our finite-size predictions for the zero-field specific heat in the low temperature region $\left(k_{B} T / J=0.10\right.$ and $\left.k_{B} T / J=0.05\right)$ are illustrated in Fig.6, performing direct diagonalization with free boundary conditions (Kamieniarz et al., 1996b). The results of the standard finite-segment calculations $(D=0$, $N \leq 14$ ), given by $\diamond$ symbols, reveal a strong size dependence. Assuming $D \neq 0$ and imposing $\left\langle\sigma_{0}^{x} \sigma_{1}^{x}\right\rangle=\overline{\left\langle\sigma_{i}^{x} \sigma_{i+1}^{x}\right\rangle}$, we find that the variation in terms of $1 / N$ is significantly reduced and the data converge towards the same asymptotic value.

We consider these results encouraging and our scheme a key issue to get reliable extrapolations of the thermodynamic quantities at temperatures lower than those considered earlier (Campana et al., 1990). 
The standard molecular field approximation is still a valuable tool for obtaining qualitative understanding of phase diagrams whenever exact solutions are impossible. An extended Ashkin-Teller model (Pawlicki, Kamieniarz and Rogiers, 1995) belongs to that class and can be expressed in terms of spin variables $s, \sigma= \pm 1$ as

$$
\mathcal{H}=-\sum_{\langle i, j\rangle}\left[J s_{i} s_{j}+J^{\prime} \sigma_{i} \sigma_{j}+J_{4}\left(s_{i} \sigma_{i}\right)\left(s_{j} \sigma_{j}\right)+J_{0}\right]-\sum_{\langle i, j\rangle}\left[K\left(s_{i}+s_{j}\right)\left(\sigma_{i} \sigma_{j}+1\right)\right]
$$

Phase diagram of the original model $(\mathrm{K}=0)$ on a two dimensional lattice has been discussed by Baxter (1982). Some renormalisation group calculations (Cachine, Drugovich de Feli and Caticha, 1989) have been undertaken to obtain a more accurate description of the diagram. Exact solutions for a limited range of interaction parameters are also available (Pearce and Seaton, 1990).

We performed a numerical molecular-field analysis of the extended model, considering a bipartite lattice and the following order parameters:

$$
\begin{aligned}
\left\langle s_{1, i}\right\rangle & =m_{1}, & & \left\langle s_{2, i}\right\rangle=m_{2}, \\
\left\langle\sigma_{1, i}\right\rangle & =n_{1}, & & \left\langle\sigma_{2, i}\right\rangle=n_{2}, \\
\left\langle s_{1, i} \sigma_{1, i}\right\rangle & =p_{1}, & & \left\langle s_{2, i} \sigma_{2, i}\right\rangle=p_{2},
\end{aligned}
$$

where integers 1 or 2 distinguish between different sublattices and (...) is a thermal average.

Results of our numerical analysis for the symmetric case $J=J^{\prime}>0$ are summarized in Fig. 7, which displays a rich temperature phase diagram. The surface of phase transitions is symmetric with respect to the plane $K / J=0$ so that we show only a few lines corresponding to $K / J<0$. Except for the areas denoted by broken lines, the critical surface consists of second order transition points. At these points only for $K=0$ all the order parameters simultaneously vanish. For $K \neq 0$, the parameters $m_{1}, m_{2}$ remain always nonzero because $K$ acts on spins as a magnetic field.

At point A two lines of tricritical points appear according to the symmetry. On the FM line the surface bifurcates. In a higher part (FMG and its symmetric counterpart for $K<0$ ) there is a jump in all the order parameters and with increasing $J_{4}$ this surface turns into a single second order transition line GC. At the point $\mathrm{H}$, the surface indicated by lines $\mathrm{c}$ begins to climb up along the line a.

For $J_{4} / J<-1$ another sheet of critical surface emerges from point $\mathrm{D}$ with two lines of tricritical points. Again, only a line for $K>0$ is indicated. Two separate sheets of critical suface are joined by a single, second order transition line $\mathrm{DH}$. 


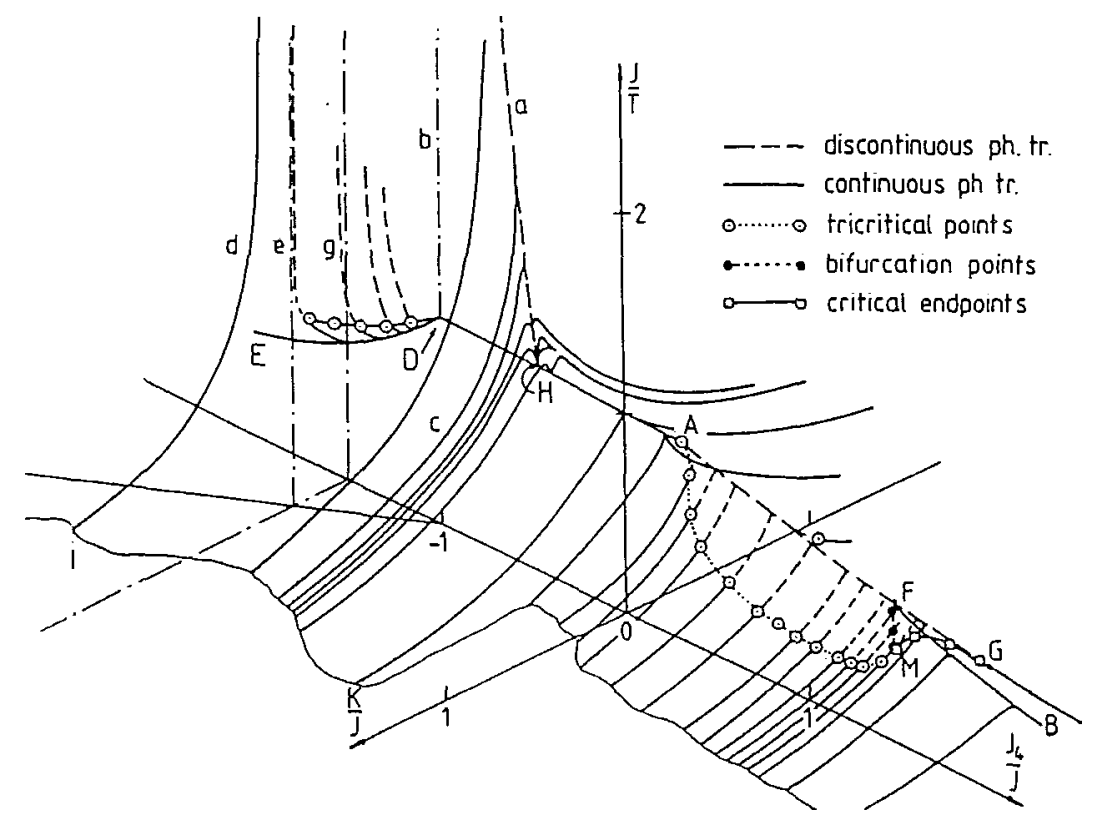

Figure 7: Phase diagram in $J / T, K / J, J_{4} / J$ variables. Lines d,e,g are in $J_{4} / J=-1.5$ plane. Lines a, b, EDHAFGC and FB are in $K=0$ plane.

\section{Results of simulations}

\subsection{3d Ising model}

The three-dimensional Ising model continues to be a subject of investigations, in particular its critical behaviour. Whereas the most accurate results traditionally came from series expansions (Liu and Fisher, 1989; Nickel and Rehr, 1990) and from the $\epsilon$-expansion (Le Guillou and Zinn-Justin, 1980), the error margins quoted in Monte Carlo based approaches are shrinking considerably (Ferrenberg and Landau, 1991; Baillie, Gupta, Hawick and Pawley, 1992). However, numerical results of such simulations are not always in a satisfactory agreement with one another. Although these differences are not large, they invite the consideration of possible sources of systematic errors such as effects due to a poor random number generator, effects introduced by histogramming methods, the use of a single row of pseudo-random numbers for a few parallel simulations or neglecting corrections to scaling in fitting procedures of simulation results.

We have simulated simple cubic Ising lattices with periodic boundary conditions and analyseed the amplitude ratio

$$
g_{L}=Q_{L}^{-1}
$$


where $Q \boldsymbol{L}$ is given by Eq.(22) and $L$ is the linear system size. The Monte Carlo data were mainly generated using the Swendsen-Wang cluster method for $K=$ 0.221653 (Blöte et al., 1993) but we also included some contributions from the largest-cluster method and Wolff algorithm (Blöte and Kamieniarz, 1994).

Table 2: Finite-size results for the universal ratio $g_{L}$ of the three-dimensional Ising model. The first column shows the finite size $L$, and the second one our Monte Carlo data with standard errors in the last decimal place between parentheses. The next column shows the length of the simulations. The fourth column lists our exact numerical transfer matrix results, and the last one contains, for comparison, part of the numerical results of Livet.

\begin{tabular}{|c|c|c|c|c|}
\hline$L$ & $g_{L}$ (present) & \# conf. & $g_{L}$ (exact) & $g_{L}$ (Livet) \\
\hline 3 & $1.49599(7)$ & $1.4 \times 10^{8}$ & 1.496016 & \\
4 & $1.51565(7)$ & $1.8 \times 10^{8}$ & 1.515605 & $1.5167(6)$ \\
5 & $1.52956(14)$ & $6.4 \times 10^{7}$ & & \\
6 & $1.54032(19)$ & $4.8 \times 10^{7}$ & & $1.5431(6)$ \\
7 & $1.5483(2)$ & $4.8 \times 10^{7}$ & & \\
8 & $1.5545(2)$ & $4.8 \times 10^{7}$ & & $1.5572(9)$ \\
9 & $1.5597(2)$ & $4.8 \times 10^{7}$ & & \\
10 & $1.5636(2)$ & $4.8 \times 10^{7}$ & & \\
11 & $1.5666(2)$ & $4.8 \times 10^{7}$ & & \\
12 & $1.5696(2)$ & $4.8 \times 10^{7}$ & & $1.5709(14)$ \\
13 & $1.5722(3)$ & $4.0 \times 10^{7}$ & & \\
14 & $1.5740(3)$ & $4.0 \times 10^{7}$ & & \\
15 & $1.5756(4)$ & $2.0 \times 10^{7}$ & & \\
16 & $1.5766(4)$ & $2.0 \times 10^{7}$ & & $1.5791(13)$ \\
18 & $1.5790(4)$ & $2.0 \times 10^{7}$ & & \\
20 & $1.5825(4)$ & $2.0 \times 10^{7}$ & & \\
22 & $1.5837(4)$ & $2.0 \times 10^{7}$ & & \\
24 & $1.5857(4)$ & $2.0 \times 10^{7}$ & & $1.5820(25)$ \\
28 & $1.5867(4)$ & $2.0 \times 10^{7}$ & & \\
32 & $1.5875(7)$ & $1.0 \times 10^{7}$ & & $1.5943(28)$ \\
\hline
\end{tabular}

The Monte Carlo data collected in Table 2 are restricted to rather small size systems $(L \leq 32)$, but have a greater statistical accuracy than other results known to us for these systems. We have analyseed our data according to

$$
g_{L}(K)=g+b L^{y_{i}}+a_{1}\left(K-K_{c}\right) L^{y_{t}}
$$

with $a_{1}=0.86$ and $y_{i}=1.59$ (Liu et al., 1989; Nickel et al, 1990; Le Guillou et al., 1980; Ferrenberg et al, 1991) in order to determine $K_{c}, \mathrm{y}_{\mathrm{i}}$ and $g$. A 
least-square fit showed that the data for $L=4$ deviate significantly, and the fits thus apply to $L \geq 5$.

We found that $g=1.609 \pm 0.004, y_{i}=-0.85 \pm 0.04$, in a good agreement with a recent series result of Nickel and Rehr (Nickel et al, 1990): $\mathrm{y}_{\mathrm{i}}=-0.83 \pm 0.05$. If we assumed a fixed value $\mathrm{y}_{\mathrm{i}}=-0.8$, as was done by Livet (1991), we would obtain $K_{c}=0.2216445$ (12) for the critical point. Including $y_{i}$ as a variable, our final estimate of the critical point is

$$
K_{c}=0.221649(4)
$$

If the critical point is indeed within these error bounds, Rosengren's conjecture (Rosengren, 1986) tanh $K_{c}=(\sqrt{5}-2) \cos (\pi / 8)$ or $\boldsymbol{K}_{c} \approx 0.2216586$ is incorrect. Our result for $K_{c}$ is about three standard errors smaller than that quoted by Landau (1994), and three or more standard errors larger than values listed by Liu et al. (1989). A comprehensive comparison is given in the review of Landau (1994).

\section{2 $2 d$ Ising-like models}

The universal critical point ratio $Q$ is a measure of the shape of the magnetization distribution $\left(\mathrm{Q}=\frac{1}{3}\right.$ for a Gaussian distribution and $Q=1$ for the long-range ordered state). Its value for square, critical Ising systems with toroidal boundary conditions was determined by Bruce (1985), and by Burkhardt and Derrida (1985) as $Q \approx \mathbf{0 . 8 6}$.

It is a very useful quantity for accurate determination of the critical points of models that are not solvable, but can be assumed to belong to the Ising universality class. The critical point of such a model can be estimated by application of fitting procedures to Monte Carlo results for $\mathrm{Q}_{L}$ near criticality.

The ratio $Q$ depends not only on the type of boundary conditions (Kamieniarz et al., 1993a). In the isotropic case, it depends also on the aspect ratio and in the anisotropic case, on the ratio of the coupling strengths in different directions (Kamieniarz et al., 1993a).

Conformal invariance has been used (Burkhardt et al., 1985) to calculate Q, but unfortunately this approach is restricted to rather special boundary conditions. On the other hand, the transfer-matrix results of Burkhardt et al. (1985) allow a rather accurate determination of $Q$ for square systems with toroidal boundary conditions: graphical extrapolation (Blote, Compagner and Hoogland, 1987) of the data in Table II of Burkhardt et al. (1985) yields $Q=0.856 \pm 0.002$. For high resolution Monte Carlo simulations, however, it is desirable to find the universal ratio $Q$ with an accuracy of more than 3 decimal places. 
Considering square $L \times L$ systems, the numerical transfer matrix estimates of $Q_{L}(L \leq 17)$ are calculated (Kamieniarz et al., 1993a) and coincide with those previously published for $L \leq 14$ (Burkhardt et al. 1985) up to all the decimal places quoted. Having obtained $\mathrm{Q}_{1}$, we have extrapolated the data using, the asymptotic formula (28) for $L \rightarrow \infty$. We have performed direct iterative fits of this expression, taking into account different numbers of terms and we estimated

$$
Q=0.856216(1)
$$

with a numerical uncertainty \pm 1 on the last decimal place, given in parenthesis.

Finally, results for $Q$ of some rectangular systems with different aspect ratios $\alpha$ are shown in Table 3. For $\alpha \leq 2$ the calculations used $L \times 3 \mathrm{~L}$ systems with $L \leq 14$ (except for $\alpha=1$, where $L \leq 15$ ) and anisotropic couplings (Kamieniarz et al., 1993a). This procedure leads to a better finite-size convergence than that using $L \times L$ systems. As a consequence, the $\alpha=1$ result in Table 3 is somewhat more accurate than that in (52). For $a>2$ the calculations used $\alpha L \times L$ systems with $L$ at least up to 11 .

Table 3: Numerical results for the universal functions $Q(\alpha)$ as well as for the coefficients $a_{i}$ in the power series $Q(\alpha)$. Estimated numerical uncertainties in the last decimal place are quoted in parentheses.

\begin{tabular}{|c|l|r|l|r|r|}
\hline$\alpha$ & \multicolumn{1}{|c|}{$Q(\alpha)$} & \multicolumn{1}{|c|}{$\alpha$} & \multicolumn{1}{|c|}{$Q(\alpha)$} & \multicolumn{1}{c|}{$a_{i}$} & \multicolumn{1}{c|}{$a$} \\
\hline 1.00 & $0.8562157(5)$ & 10 & $0.441345(1)$ & 0 & 0.66965061 \\
1.25 & $0.851947(1)$ & 15 & $0.3987253(3)$ & 1 & 0.32377692 \\
1.50 & $0.8415515(7)$ & 20 & $0.3800929(2)$ & 2 & -0.15701679 \\
1.75 & $0.827049(1)$ & 30 & $0.363112(8)$ & 3 & -0.01878447 \\
2.00 & $0.809678(3)$ & 40 & $0.355179(6)$ & 4 & 0.09094136 \\
3.00 & $0.728090(3)$ & 50 & $0.350584(5)$ & 5 & -0.11922324 \\
4.00 & $0.650069(3)$ & 60 & $0.347586(4)$ & 6 & 0.06618100 \\
5.00 & $0.587126(2)$ & 70 & $0.345476(3)$ & 7 & 0.09431846 \\
6.00 & $0.539396(2)$ & 80 & $0.343910(3)$ & 8 & -0.12230677 \\
7.00 & $0.503811(1)$ & 90 & $0.342702(2)$ & 9 & -0.00847109 \\
8.00 & $0.477176(1)$ & 100 & $0.341742(1)$ & 10 & 0.04733566 \\
9.00 & $0.456964(1)$ & $\infty$ & $0.333333(3)$ & 11 & -0.01018046 \\
\hline
\end{tabular}

Within a margin of $3 \times 10^{-5}$, our results for $Q(\alpha)$ are reproduced by the expression

$$
Q(\alpha)=\sum_{i=0}^{11} a_{i} A^{i}
$$


where $A=4 /(\alpha+1 / \alpha)-1$, and the coefficients $\mathrm{a}_{i^{-}}$are quoted in the last column of Table 3. Also, using power law fits in $1 / \alpha$ to the data for large aspect ratios, we have estimated that:

$$
\lim _{\alpha \rightarrow \infty} Q(\alpha)=0.333333(3)
$$

which agrees with the value $\frac{1}{3}$ for the Gaussian distribution describing linear systems.

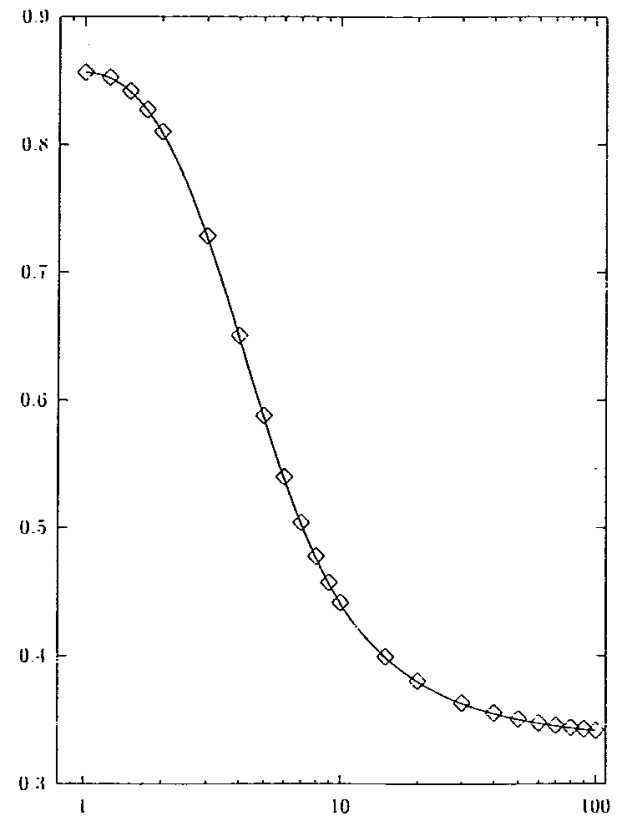

Figure 8: The universal function $Q(\alpha)$ as a function of the aspect ratios $\alpha$ in the extended interval $1 \leq \alpha \leq 100$, plotted on a semi-logarithmic scale. The curve represents fitting polynomial and interpolates through the discrete data shown by the symbol $\diamond$.

The critical point invariant $Q$ is plotted in Fig. 8 on a semi-logarithmic scale versus the aspect ratio $\alpha$. The smooth curve represents the polynomial fitting (53).

Apart from the calculation of the ratio $Q$ for the Ising model, we addressed the problem of the universality class for the non-interacting hard-square lattice-gas model (Baxter, Enting and Tsang, 1980). The absence or presence of a latticegas particle at site $i$ is expressed by a variable $\sigma_{i}=0$ or $\sigma_{i}=1$, respectively. Denoting the activity of the gas particles as the partition sum for the non- 
interacting hard-square model (Baxter et al., 1980) is

$$
Z=\sum_{\sigma_{i}} z^{\Sigma_{i} \sigma_{i}} \prod_{<i, j>}\left(1-\sigma_{i} \sigma_{j}\right)
$$

where the product is over all pairs of nearest-neighbour sites, and guarantees that configurations with interpenetrating particles do not contribute to $Z$.

In absence of an exact solution, Baxter et al. (1980) applied series expansion techniques to determine the critical exponents of this model. They found that the specific heat exponent $\alpha^{\prime}=0.09 \pm 0.05$ which was different from the exactly known value of the two-dimensional Ising model $\alpha=0$. Subsequent analyses of the finite-size scaling behaviour of the temperature derivative of the correlation length (Wood and Goldfinch, 1980; Racz, 1980) did not show significant deviations from the Ising universality. However, we found the result by Baxter et al. (1980) sufficiently challenging to find more compelling numerical evidence that the hard-square model is inside the Ising universality class.

On the basis of the result for the critical activity (Blöte and Wu, 1990)

$$
z_{c}=3.796255174(3)
$$

where the translational symmetry of the model (54) is spontaneously broken, the series expansions evaluated by Baxter et al. (1980) can be reanalysed.

Thus we consider (Kamieniarz and Blöte, 1993b) an expansion of the order parameter (i.e. the staggered density) $R$ in terms of the high-density parameter $x=1 / z$. From the ratio analysis of $R \sim^{8}(x)$, using $z_{c}$ and the coefficients listed in Table II of Baxter et al. (1980) we have estimated that the critical exponent $\beta=0.1249(1)$, in agreement with Ising universality.

The subsequent Dlog Padé analysis of the series $R(x)$ (as that in Table I of Baxter et al. (1980)) and the smooth dependence (Fig. 9) of the residues of $\frac{d}{d x} \ln \_\mathrm{R}(\mathrm{a}:)$ on the distance of the corresponding poles from the critical value $x_{c}=0.263417488$ indicate that $\beta$ does not differ from $1 / 8$ by more than a few times $10^{5}$.

To calculate $\alpha$, the second derivative $\rho^{(2)} \equiv \frac{d^{2}}{d x^{2}} \rho^{\prime}$ of the lattice-gas density series $\rho^{\prime}$ is chosen which is more singular than $\rho$ and can be found from the series $\rho^{\prime}=1-2 \rho=\sum_{n=1}^{\infty} \rho_{n} x^{n}$, where the coefficients $\rho_{n}$ are determined by those given in Appendix B of Baxter et al. (1980). Performing the Dlog analysis of the $\rho^{(2)}$ series (Kamieniarz et al., 1993b), we estimate $\alpha^{\prime}=-0.1(1)$ which indicates that $\alpha^{\prime}$ may be smaller than the result obtained by Baxter et al. (1980) from the $R\left(\rho^{\prime}\right)$ series. The dependence of the $\alpha^{\prime}$ estimates on the distance from the critical point $x_{c}$ is shown in Fig. 10.

In Tables 4 and 5 our additional differential approximant analyses (Fisher and Au-Yang, 1979) of i ${ }^{1}\left(\rho^{\prime}\right)$ and $\rho(x)$ series are shown. The columns denoted 


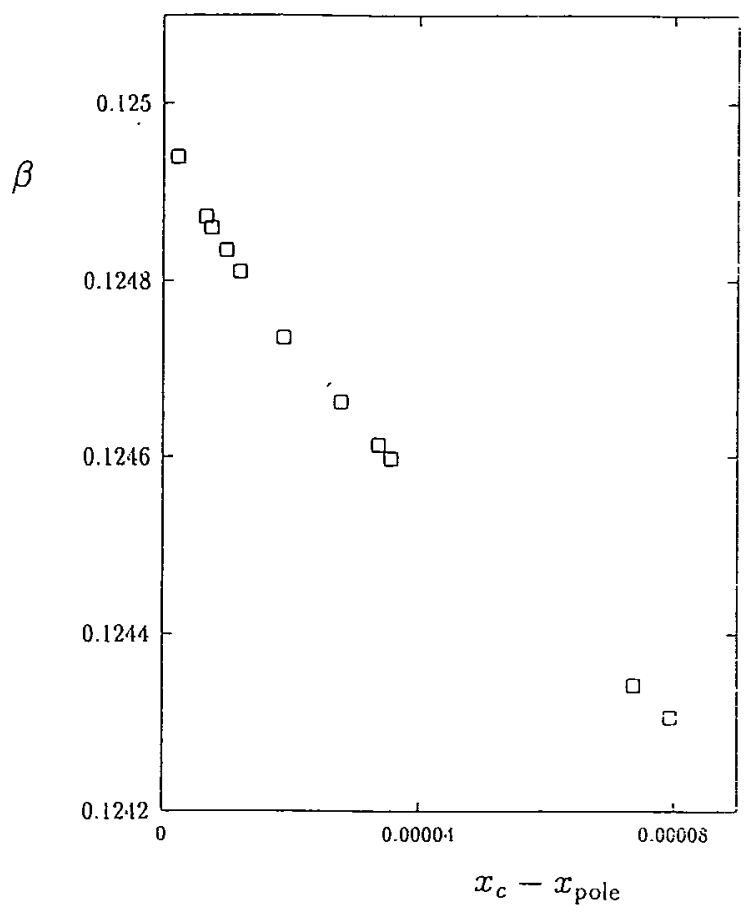

Figure 9: The residues of the D-log Pade approximants of the series $R(x)$ versus the deviations of the corresponding poles from the critical value $x_{c}$

$N, L, M$ display the degrees of the corresponding polynomials. $\overline{\rho_{c}^{\prime}}$ and $\overline{x_{c}}$ represent the calculated unbiased critical parameters $\rho_{c}^{\prime}$ and $x_{c}$ which are obtained from the given approximants. Subscripts $u$ and $b$ denote the unbiased and biased (choosing $\rho_{c}^{\prime}=0.264514, x_{c}-0.2634175$ ) estimates of the corresponding critical

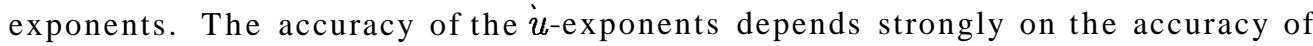
the estimates $\overline{\rho_{c}^{\prime}}$ and $\overline{x_{c}}$. However, the 6 -exponents deviate from the Ising values within a margin of 0.002 and 0.005 for $\beta /\left(1-\alpha^{\prime}\right)$ and $\alpha^{\prime}$, respectively (where we disregard the estimate for the approximant $[6,5,10])$.

In addition, we consider the lattice-gas model (54) on $L \times L$ and $L \times \infty$ square lattices with periodic boundary conditions. To enable the introduction of two sublattices in a checkerboard-like fashion, $L$ is restricted to be even. We use the transfer matrix technique (Kamieniarz et al., 1993b) in order to calculate the partition sum (54), as well as some of its derivatives.

A finite-size analysis is used to determine yh and yt. Other exponents follow from these by means of scaling. Here, the main interest is in the value of $y_{t}$ in view of its relevance for expressed by $=2-d / y_{t}$. The assumptions 


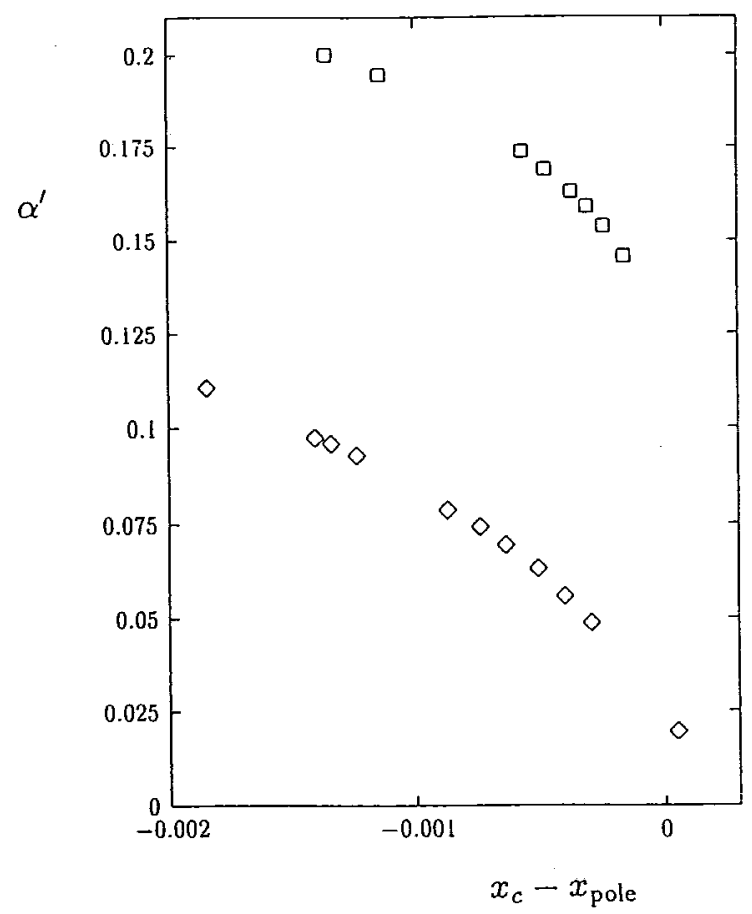

Figure 10: The dependence of the $\alpha^{\prime}$ estimates found from the D-log Padé approximants on the deviations of the poles from the critical point. The present data are shown versus $x_{c}-x_{\text {pole }}$ by $\diamond$, and those of Baxter et al. versus $\rho_{c}-\rho_{\text {pole }}$ by $\square$.

involved amount to the analyticity of renormalisation transformations employing an additional finite-size field (Barber, 1983) $1 / \mathrm{L}$ with exponent $\mathrm{y}_{\mathrm{L}}=1$.

We have determined finite-size data for several quantities: the density $\rho_{L}$ of the lattice gas particles, the specific heat $c_{L}$, the staggered susceptibility $X_{L}$, and the temperature derivative $g_{i}^{\prime}(L)$ of the inverse magnetic correlation length. They are expected (Kamieniarz et al., 1993b) to fulfil the finite-size scaling relations

$$
\begin{aligned}
& \rho_{L} \simeq \rho_{c}+L^{y_{t}-d}\left(a_{1}+a_{2} L^{-p}\right), c_{L} \simeq L^{2 y_{t}-d}\left(b_{1}+b_{2} L^{-q}\right), \\
& \chi_{L} \simeq L^{2 y_{h}-d}\left(c_{1}+c_{2} L^{-r}\right), g_{i}^{\prime}(L) \simeq L^{y_{t}-1}\left(d_{1}+d_{2} L^{-w}\right),
\end{aligned}
$$

where $d=2$ is the dimensionality and the terms with exponents $p, r, q, w$ represent corrections to scaling. As an example, we show the results for a square system in Table 6 . The scaling behaviour of all these data is in an accurate agreement (Kamieniarz et al., 1993b) with Ising universal exponents.

Another approach intimately related with renormalisation was made using the hypothesis of conformal invariance (Cardy, 1987). The temperature and 
Table 4: The differential approximant analysis of the series $R^{-1}\left(\rho^{\prime}\right)$.

\begin{tabular}{|rrr|c|c|c|}
\hline $\mathrm{N}$ & $\mathrm{L}$ & $\mathrm{M}$ & $\bar{\rho}_{c}^{\prime}$ & $\left(\frac{\bar{\beta}}{1-\alpha^{\prime}}\right)_{u}$ & $\left(\frac{\beta}{1-\alpha^{\prime}}\right)_{b}$ \\
\hline 7 & 7 & 7 & 0.264465 & 0.118388 & 0.126657 \\
8 & 5 & 8 & 0.264488 & 0.122358 & 0.126509 \\
7 & 6 & 8 & 0.264483 & 0.121542 & 0.126559 \\
8 & 4 & 9 & 0.264535 & 0.129885 & 0.126066 \\
6 & 7 & 8 & 0.263892 & 0.018301 & 0.126448 \\
7 & 5 & 9 & 0.264491 & 0.122845 & 0.126751 \\
5 & 8 & 8 & 0.264692 & 0.157027 & 0.125629 \\
6 & 6 & 9 & 0.264552 & 0.132852 & 0.126476 \\
5 & 7 & 9 & 0.264583 & 0.137947 & 0.126610 \\
6 & 5 & 10 & 0.264471 & 0.119259 & 0.145598 \\
4 & 8 & 9 & 0.264060 & 0.041619 & 0.123054 \\
5 & 6 & 10 & 0.264597 & 0.140181 & 0.124088 \\
\hline
\end{tabular}

magnetic exponents were determined using the relation (29) between exponents and the finite-size amplitude of the associated correlation lengths for the infinite strips. The ensuing results (Kamieniarz et al., 1993b)

$$
y_{t}=1.000000(1), y_{h}=1.875000(1)
$$

are in precise agreement with the exactly known Ising values $y_{h}=15 / 8$ and $y_{t}$

$$
=
$$

In addition to these results for the exponents, we have also found the critical density

$$
2 \times p_{c}=0.73548600(1)
$$

and the universal critical point ratio (Kamieniarz et al., 1993a)

$$
Q=\left\langle R^{2}\right\rangle^{2} /<R^{4}>=0.85625(5),
$$

the latter in agreement with the corresponding Ising value (52) for ferromagnetic Ising models with periodic boundaries of the square symmetry.

\subsection{Quasi one-dimensional magnets}

Quasi-one-dimensional magnets have attracted a great deal of interest due to nonlinear spin dynamic effects and the recognition of the role of the spin value in the ground state critical properties. An extensive account of the theoretical and experimental results can be found elsewhere (Mikeska and Steiner, 1991; Halperin, 1992). 
Table 5: The differential approximant analysis of the series $\rho(x)$.

\begin{tabular}{|rrr|c|c|c|}
\hline $\mathrm{N}$ & $\overline{\mathrm{L}}$ & $\cdot \mathrm{M}$ & $\overline{x_{c}}$ & $\left(1-\alpha^{\prime}\right)_{u}$ & $\left(1-\alpha^{\prime}\right)_{b}$ \\
\hline 7 & 6 & 7 & 0.263490 & 0.985605 & 0.999678 \\
8 & 4 & 8 & 0.263516 & 0.981007 & 0.994742 \\
6 & 7 & 7 & 0.263417 & 0.999936 & 0.999765 \\
7 & 5 & 8 & 0.263608 & 0.963034 & 0.998233 \\
6 & 6 & 8 & 0.263422 & 0.998815 & 0.999693 \\
7 & 4 & 9 & 0.263491 & 0.984604 & 0.995466 \\
5 & 7 & 8 & 0.263417 & 0.999779 & 0.999766 \\
6 & 5 & 9 & 0.263549 & 0.973867 & 0.100050 \\
4 & 8 & 8 & 0.263415 & 0.999955 & 0.999424 \\
5 & 6 & 9 & 0.263494 & 0.984681 & 0.999637 \\
4 & 7 & 9 & 0.263330 & 1.017277 & 1.000238 \\
5 & 5 & 10 & 0.263543 & 0.975050 & 0.995529 \\
\hline
\end{tabular}

First, we recall here the zero-field specific heat results for $C H A B$ (i.e. the spin $S=1 / 2$ compound $\mathrm{C}_{6} \mathrm{H}_{11} \mathrm{NH}_{3} \mathrm{CuBr}_{3}$ ). In Fig. 11 the dashed curve represents the experimental data, whereas our numerical estimates (Campana et al., 1990) are given by solid circles. The vertical intercepts show the numerical uncertainties for $N \leq 12$ in our extrapolation procedure (34).

In Fig. 12 the $C H A B$ high-field specific-heat data are depicted. The small symbols denoted by crosses and open and solid circles show the experimental excess specific heat $\triangle C(T, B)$. The bigger crosses and solid circles with the error bars represent our estimates found from the polynomial fitting (32). We

Table 6: The finite-size data $2 \rho_{L}, c_{L}$ and $\chi_{L}$ calculated numerically for square systems.

\begin{tabular}{|r|c|c|c|}
\hline $\mathrm{L}$ & $2 \rho_{L}$ & $c_{L}$ & $\chi_{L}$ \\
\hline 2 & 0.8090898056421140 & 0.1402349991566989 & 0.724743813172343 \\
4 & 0.7749712801688777 & 0.1922474459514767 & 2.447647203710980 \\
6 & 0.7616725216302425 & 0.2246002865877822 & 4.978297322277479 \\
8 & 0.7550639427551426 & 0.2477057485415912 & 8.241664994051973 \\
10 & 0.7511214217544006 & 0.2656570381718074 & 12.18609189883877 \\
12 & 0.7485021560614531 & 0.2803252992432338 & 16.77381425559746 \\
14 & 0.7466353591805462 & 0.2927219631820310 & 21.97568172323616 \\
16 & 0.7452373256688473 & 0.3034545757008545 & 27.76817664548804 \\
\hline
\end{tabular}




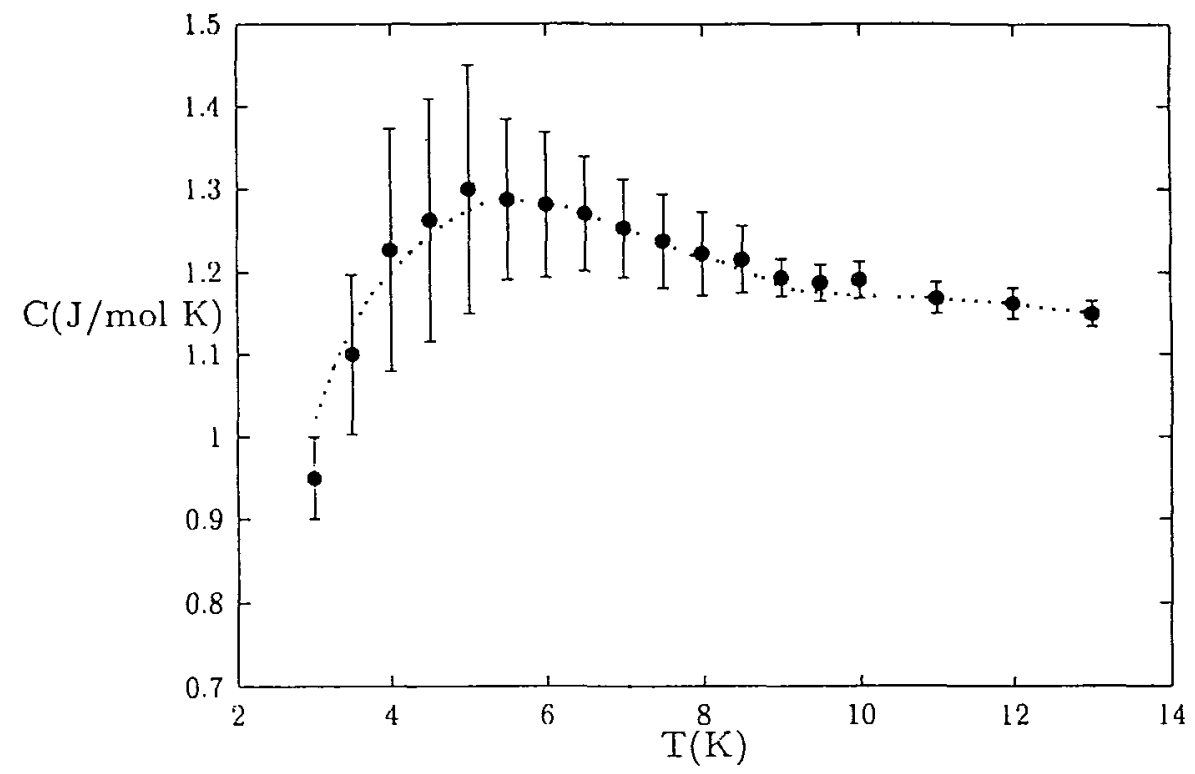

Figure 11: Zero-field specific heat of $\mathrm{CHAB}$. The dashed curve represents the experimental results. Present theoretical results are shown by solid circles with error bars.

encountered a good convergence of the extrapolations for temperatures $T \geq \mathbf{1 0 K}$, including the position of the peak for $B=3 \mathrm{~T}$ and we found agreement with QTM predictions (Kopinga et al., 1990).

The zero-field specific heat is also calculated for $\mathrm{C}_{6} \mathrm{H}_{11} \mathrm{NH}_{3} \mathrm{CuCl} l_{3}$ (the $\mathrm{S}=\frac{1}{2}$ compound abbreviated as $C H A C$ ) and is shown in Fig. 13. From the ferromagnetic resonance experiment it has been established that $C H A C$ can be described be the anisotropic Heisenberg Hamiltonian with $J_{x} / k_{B}=45.52 K, \quad J_{y} / k_{B}=$ $44.99 K$ and $J_{z} / K_{B}=44.49 K$. In Fig. 13 the experimental curve is drawn in the solid line with a sharp peak corresponding to the phase transition point to the three-dimensional (3D) ordering. Our results are marked by the circles and are consistent with the experimental findings down to $2 K$ above the transition temperature. An additional fitting procedure for $C H A C$ was carried out by Kopinga, Delica, Leschke and Riedel (1993).

A considerable amount of experiments has been performed (Mikeska et al, 1991) on $\mathrm{CSNiF}_{3}$, the spin $\mathrm{S}=1$ quasi-one-dimensional ferromagnet. The microscopic parameters

$$
J / k_{B}=23.6 K, \quad A / k_{B}=9 K, \quad g=2.4
$$

of the Hamiltonian describing $\mathrm{CsNiF}_{3}$ were first determined (Steiner, Villain and Windsor, 1976) by fitting the observed energy spectra to the theoretical magnon 


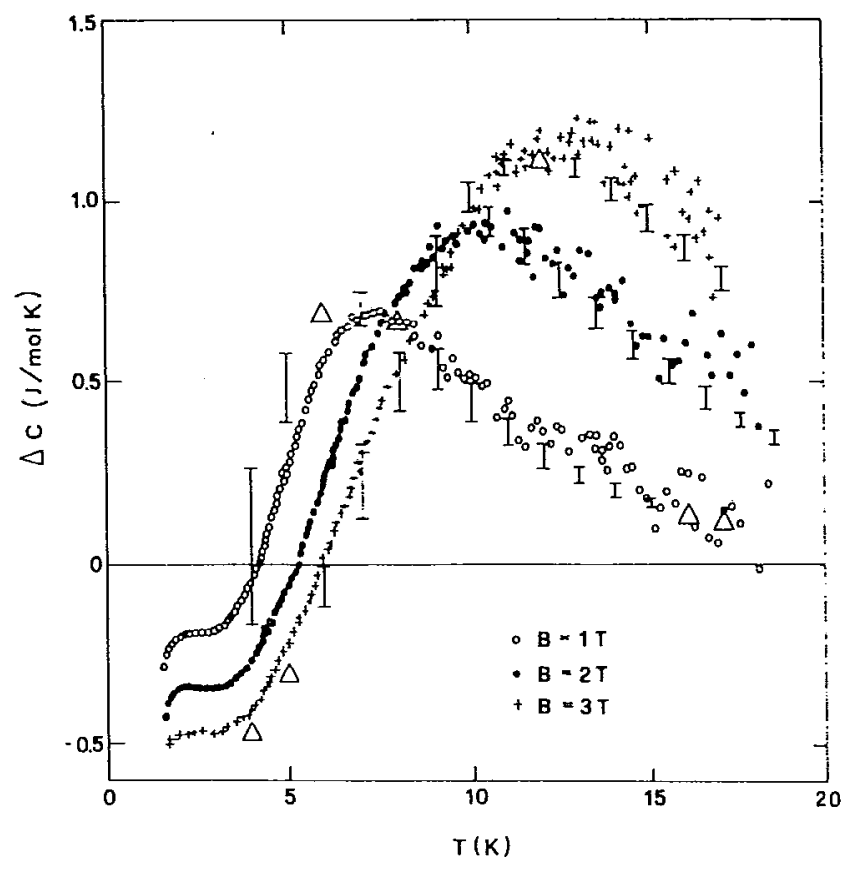

Figure 12: Excess specific heat $\Delta C(T, B)=C(T, B)-C(T, 0)$ of CHAB for $B=1,2$, and $3 T$. Open circles, solid circles, and small crosses represent the experimental data. Bigger crosses and points with error bars illustrate present estimates.

dispersion relation. Due to the discrepances between the theoretical predictions and the observed static properties evidenced in numerical calculations, new fitting procedures were carried out by Delica, de Jonge, Kopinga, Leschke and Mikeska (1991) and Campana et al. (1992a) which led to the following sets of the parameters

$$
J / k_{B}=25 K, \quad A / k_{B}=7.7 K, \quad g=2.1
$$

and

$$
J / k_{B}=20.5 K, \quad A / k_{B}=8.71 K, \quad g_{\|}=2.23, \quad g_{\perp}=2.28
$$

respectively. In both numerical procedures the values of $g$ were fixed and only $J$ and $A$ were subject to variation.

We found the competing predictions (55), (56), (57) together with a new SCHA approach (Cuccoli, Tognetti, Verrucchi and Vaia, 1992) challenging enough to start new large-scale simulations (Campana et al., 1996) by recourse to the finite-size and QTM calculations. As the sets (55), (56), (57) were found from different fitting procedures and no significant experimental results have been 


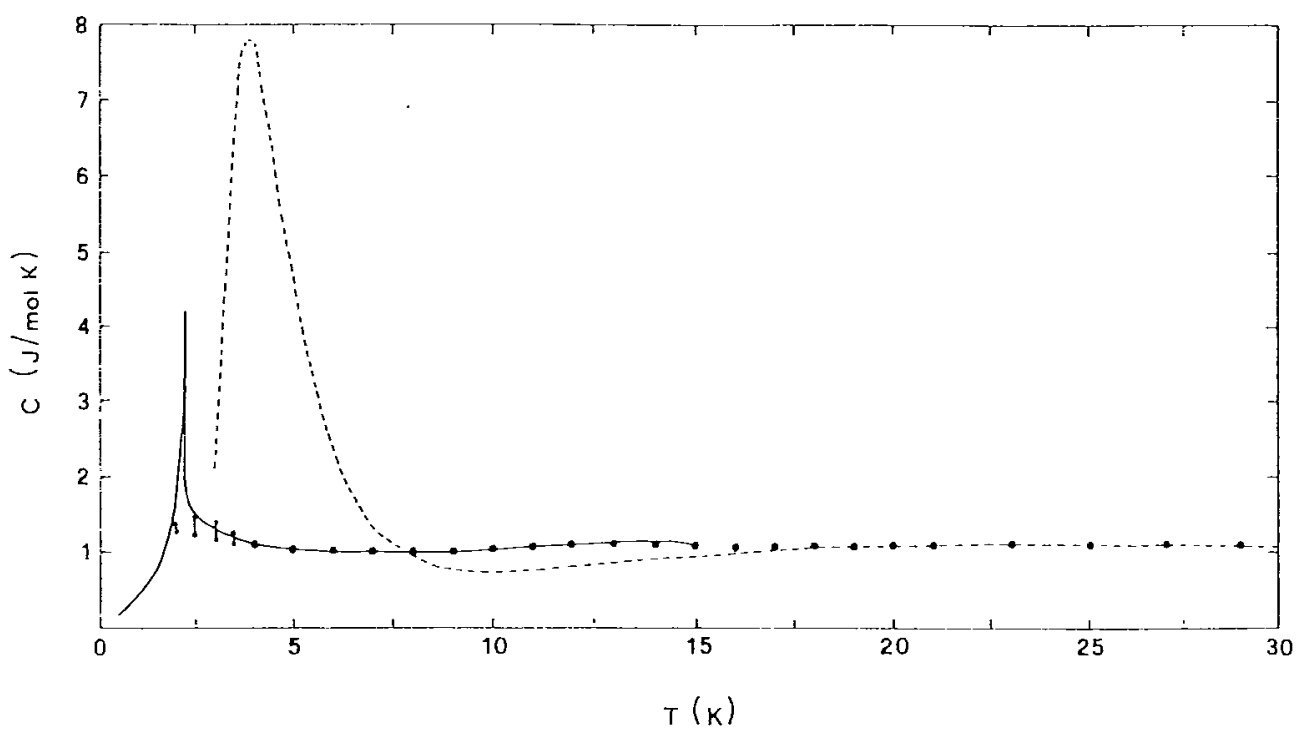

Figure 13: Zero-field specific heat of CHAC. The experimental data are plotted by the continous line and our estimates are displayed by the symbols.

reported (to our knowledge) in the meantime, so that we compared the predictions following from these values of the parameters and we determined within the unique technique the optimum set (57) with respect to static properties of $\mathrm{CsNiF}_{3}$.

We start presentation of our new results (Campana et al., 1996) from the zero-field longitudinal susceptibility $\chi_{\|}$. This quantity displays a maximum in low temperatures which is sensitive to the choice of parameters. Our numerical data are given in Fig. 14 for sets (57), (56), (55), respectively and the results of measurements (Dupas and Renard, 1977) are depicted by full circles. For set (57) found in (Campana et al., 1992a), the agreement is excellent whereas some deviations of the order of 5-6\% occur (Fig. 15) near the maximum if set (56) is used. Well pronounced discrepancy between experiment and simulations for set (55) appears in the entire temperature region.

The zero-field perpendicular susceptibility $\chi_{\perp}$ data are shown in Fig. 15 16 for the same parameters as before and using the same symbols as in Fig. 14. In addition, dotted curve in Fig. 16 illustrates the predictions of Cuccoli et al. (1992) The results of our simulations for $\chi_{\perp}$ show systematic deviations with respect to the experimental data, of the order of $2 \%$, irrespective of the parameter values. The values (56), (57) lead to an underestimation, whereas those in (55), to an overestimation. We also note that the theoretical SCHA 


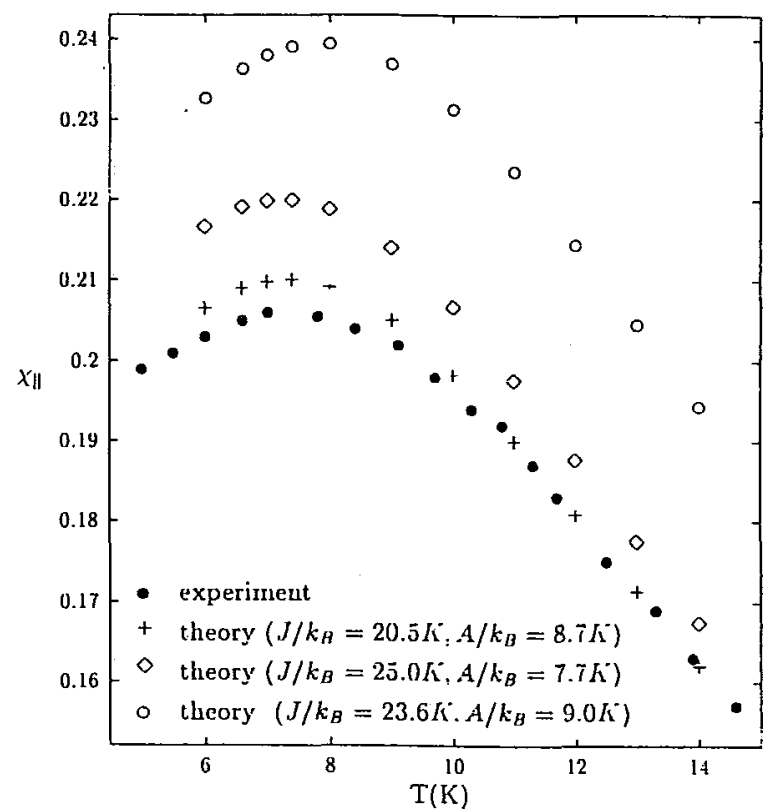

Figure 14: The temperature dependence of $\chi_{\|}$in molar units. The symbols and the parameters are specified in the label.

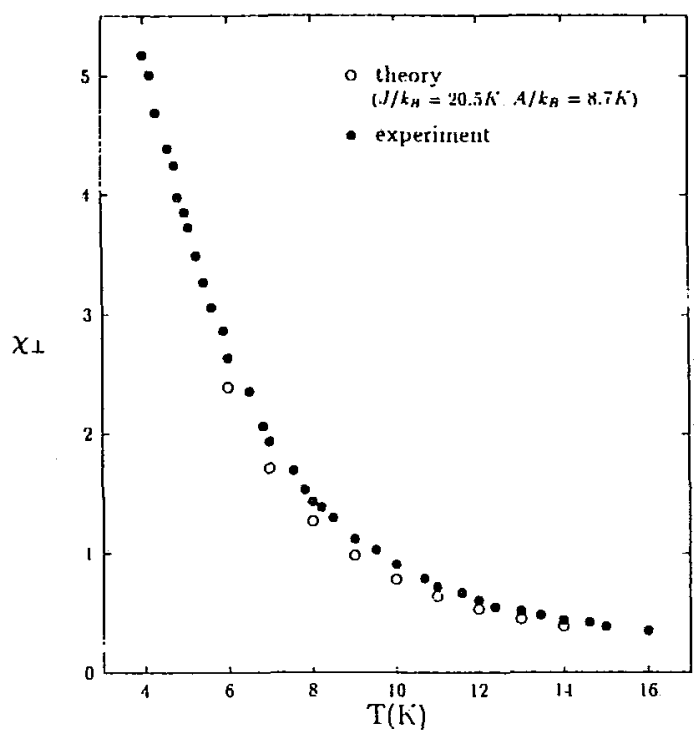

Figure 15: The temperature dependence of the molar $\chi_{\perp}$. The symbols $\bullet$ and $\circ$ represent the experimental and numerical data, respectively. 


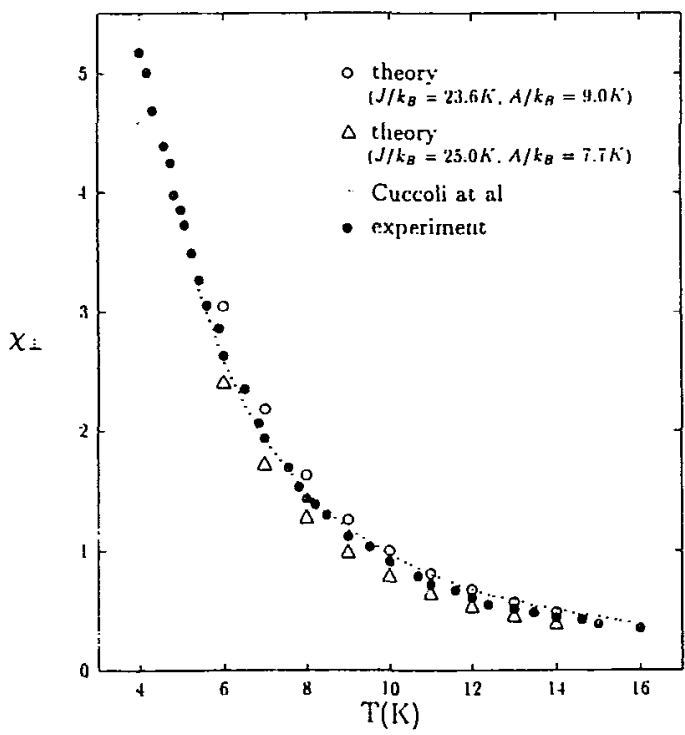

Figure 16: The temperature dependence of the molar $\chi_{\perp}$. Notation as in Fig. 15.

curve in Fig. 16 does not coincide with our predictions within the error of our simulations.

In Fig. 17 we present the excess specific-heat data in the field $5 k G s$. The corresponding experimental data (Ramirez and Wolf, 1985) are shown by full circles and our results by $\diamond, \square ; \triangle$ symbols for parameter (55), (56), (57), respectively. We also plot by the dotted curves the theoretical SCHA predictions as reported in (Cuccoli et al., 1992). Our findings for the excess specific-heat nearly coincide for parameters (56) and (57). We do not confirm the quantitative agreement with experiment for parameters (55), although the deviations between our results and those of SCHA do not exceed $20 \%$.

In conclusion, the best agreement with static properties of $\mathrm{CsNiF}_{3}$ is reached for the parameter set (57).

For the antiferromagnetic Haldane-gap system $\mathrm{CsNiCl}_{3}$, it has been established from susceptibility and specific heat measurements (de Jongh and Miedema, 1974; Moses, Ehrenfreud, Makovsky and Shechter, 1977) that $-J / k_{B}=$ $26-27 \mathrm{~K}$. However, neutron-scattering experiments (Morra, Armstrong, Buyers and Hirakawa, 1988), performed in order to test the Haldane conjecture, have led to a somewhat higher value $J / k_{B}--33.2 \mathrm{~K}$.

From our finite-size calculations (Campana, Caramico D'Auria, Esposito, Esposito and Kamieniarz, 1992b) we found the following set of parameters:

$$
J / k_{B}=-27 \pm 3 K, \quad D / J=0.05, \quad g=2.23,
$$




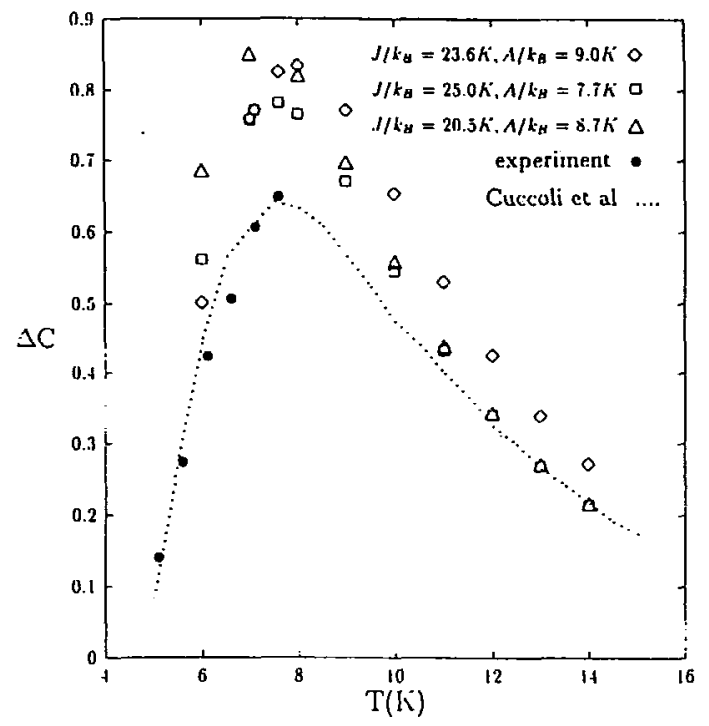

Figure 17: The temperature dependence of the excess specific heat in molar units in the applied field $B=5 \mathrm{kGs}$. The experimental data are given by the full circles. The numerical data for different parameters are presented by the symbols explained in the labels. The SCHA predictions are plotted by the dotted curve.

giving the best fit to the static measurements. These values confirm the previous findings from the static measurements (de Jongh et al., 1974; Moses et al, 1977). In particular, our $g$ value agrees very well with the one found from the ESR experiment (Achiwa, 1969). The slight anisotropy in the experimental susceptibility data can be accounted for by a small anisotropy in $\mathrm{g}$.

The numerical results (Campana et al., 1992b) for the zero-field specific heat of $\mathrm{CsNiCl}_{3}$ are represented in Fig. 18 as the solid line, whereas the dotted line gives the numerical results for $\mathrm{J} / \mathrm{k}_{\mathrm{B}}=-33.2 \mathrm{~K}$. The corresponding experimental data are represented by the circles.

In Fig. 19 we plot our numerical predictions for the parallel susceptibility $\chi_{\|}$(to aviod an overlap, we skip our estimates for $\chi_{\perp}$ ). The symbols represent the experimental data: the open circles refer to $\chi_{\|}$and the triangles to $\chi_{\perp}$. The dashed curve represents our results for $\chi_{\|}$, using the value $\mathrm{J} / \mathrm{k}_{\mathrm{B}}=-33.2 \mathrm{~K}$, which underestimate the expermental data and deviate well above the uncertainties in our extrapolations, denoted by the vertical error bars. These findings have been confirmed by our recent QTM calculations (Kamieniarz et al., 1996a).

The Haldane-gap systems attract a lot of interest (Halperin, 1992; Ramirez, Cheong and Kaplan, 1994) and new antiferromagnetic $S=1$ chains are synthetized (Gadet, Verdagner, Briois, Glezis, Renard, Beauoillain, Chappert, Goto, 


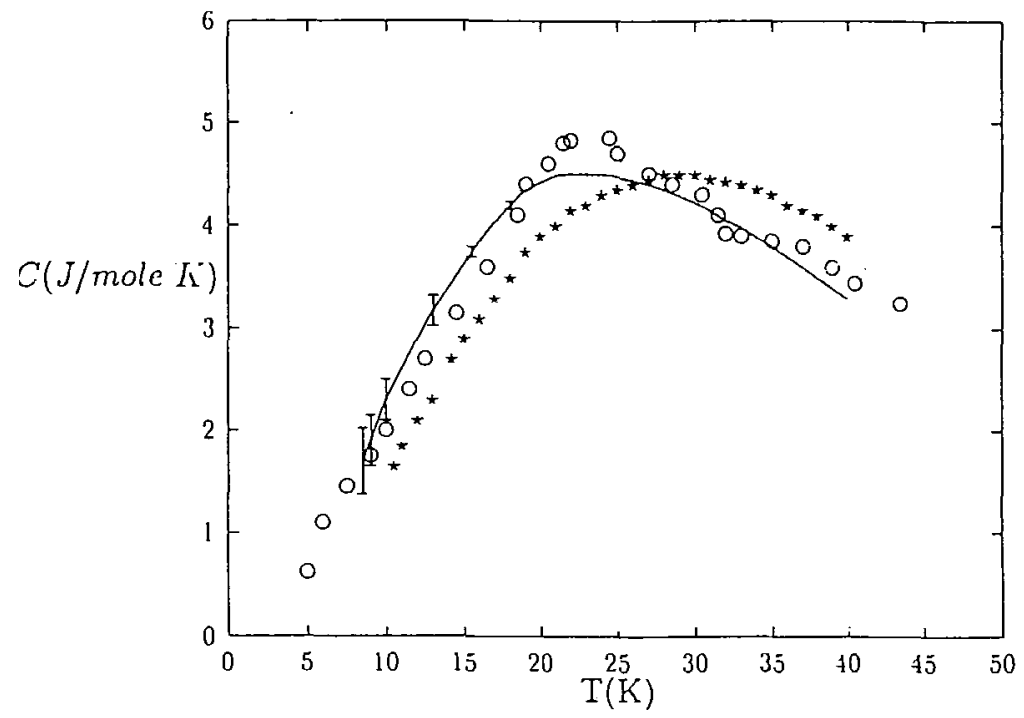

Figure 18: Specific heat of $\mathrm{CsNiCl}_{3}$. The open circles refer to experimental data. The finite-chain results for $\mathbf{J} / \mathbf{k}_{\mathrm{B}}=-27 K$ and $D / J=\mathbf{0 . 0 5}$ are drawn by the solid line with error bars. The dotted line refers to $J / k_{B}=\mathbf{- 3 3 . 2} K$.

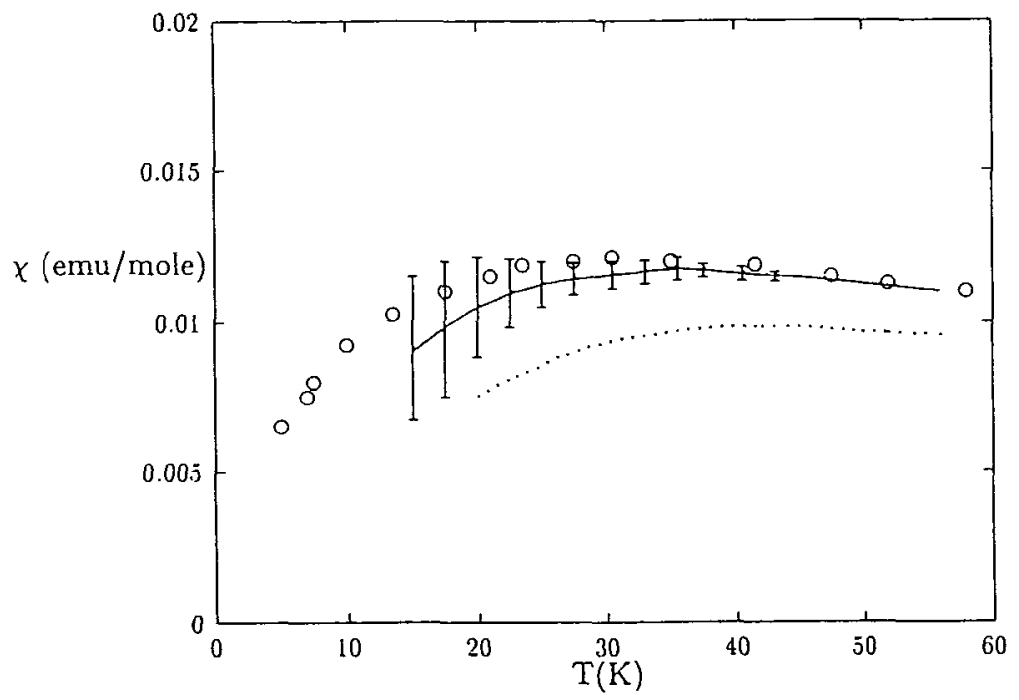

Figure 19: Susceptibility of $\mathrm{CsNiCl}_{3}$. Solid line: numerical data for $J / k_{B}=-27 \mathrm{~K}$ and $D / J=\mathbf{0 . 0 5}$. Dashed line: our data for $J / k_{B}=\mathbf{- 3 3 . 2} K$. Errors bars are shown by the intercepts. The symbols report the experimental data: the circles ${ }$, and the triangles $\chi \perp$. 


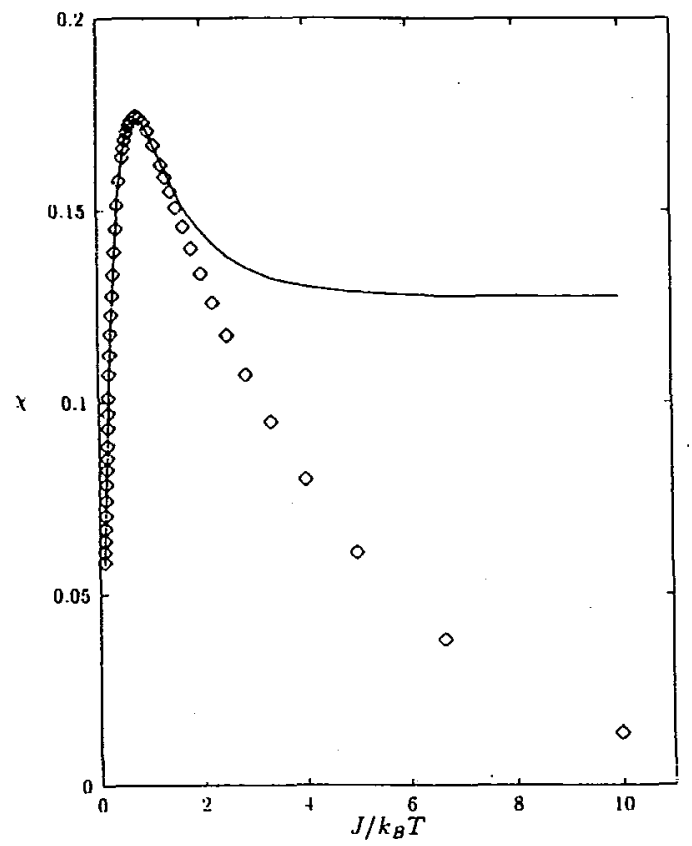

Figure 20: The temperature dependence of the isotropic susceptibility for the $5=1$ antiferromagnetic Heise.nberg chain. The symbols illustrate the numerical data and the curve represents the high-temperature expansion results.

Le Dang and Veillet, 1991; Darriet and Regnault, 1993). To help interpret the susceptibility measurements we calculated within QTM (Kamieniarz et al., 1996a) the temperature dependence of the susceptibility in the isotropic limit. Our results are shown in Fig. 20 by the $\diamond$ symbols. So far only high-temperature series expansion results of Weng (Weng, 1968) have been available and commonly used. The latter are drawn by the continuous line in Fig. 20.

\subsection{Spin-dynamics for the classical anisotropic chain}

Following Gerling et al. (1990), we have interpreted results of our simulations for the $x$ and $y$ polarizations of the neutron scattering laws for $\mathrm{CsNiF}_{3}$ by recourse to the sine-Gordon theory (Allroth and Mikeska, 1981). The sine-Gordon theory restricts spins to the XY-plane, but on general grounds (Kakurai, Steiner and Dorner, 1990) we expect to see out-of-plane fluctuations which at least give rise to a single spin-wave peak $S_{z}^{s w}(q, \omega)$ with the Lorentzian line shape adopted.

We considered (Grille et al, 1992) the spins as three-dimensional vectors of 


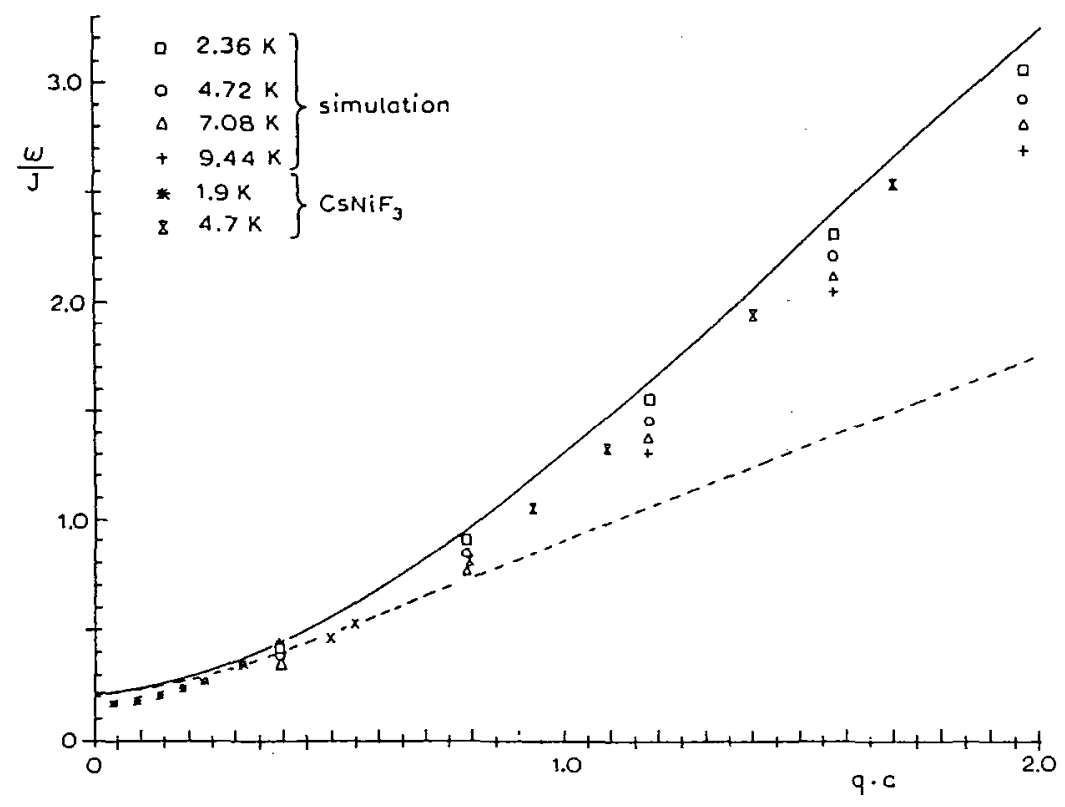

Figure 21: The spin-wave dispersion The temperatures for the simulation results (the magnetic field $B=1 \quad T$ and the anisotropy $A / k s=9-0 K$ ) and the experimental results are given in the label. A harmonic approximation and the sine-Gordon result are plotted by the solid line and the dashed line, respectively.

unit length and we chose

$$
J / k_{B}=23.6 K, A / k_{B}=9.0 K \text { or } A / k_{B}=4.5 K, g=2.25
$$

Typically, the neutron scattering laws found here are similar to those for the XY model (Gerling et al., 1990), although the interpretation is more difficult and not always unique. $S_{z}(q, \omega)$ consists of a single narrow spin-wave peak, whereas $S_{x}(q, \omega)$ and $S_{y}(q, \omega)$ develop a double-peak structure.

The fitting procedure, similar to that of Gerling et al. (1990), leads to the following predictions (Grille et al., 1992). There exist out-of-plane fluctuations yielding well-defined spin-wave excitations. The spin-wave dispersion found from $S_{y}(q, \omega)$ is presented in Fig. 21 for $A / k_{B}=9 K$ and $B=1 T$. Our results are reported by the symbols defined in Fig. 21. The experimental data, reported by asterisks, are taken after Steiner, Kakurai and Kjems (1983) in the region $q a \leq 0.4$ whereas for $q a \geq 0.4$ we have interpolated the results of Steiner and Kjems (1977). The continuous curve is a harmonic approximation and the dashed line is the result of the sine-Gordon model. For low temperatures and small wave 

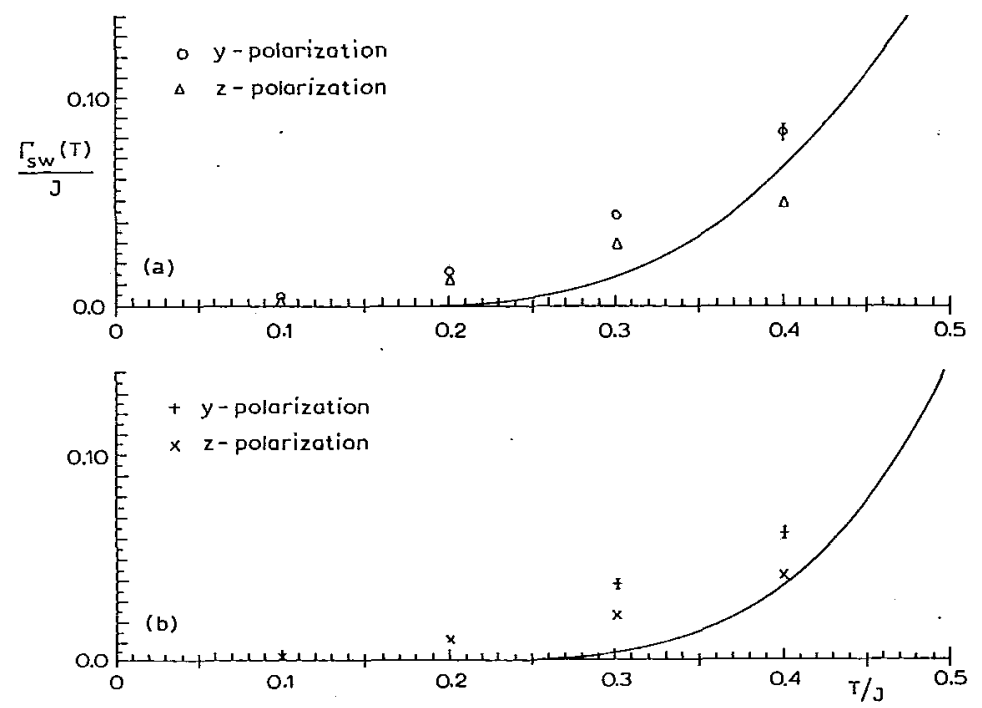

Figure 22: The $q$-dependence of the spin-wave line widths $\Gamma_{s w}$. The temperatures are as indicated and the magnetic field $B=1 T$ and the anisotropy $A / k_{B}=9.0 \mathrm{~K}$.

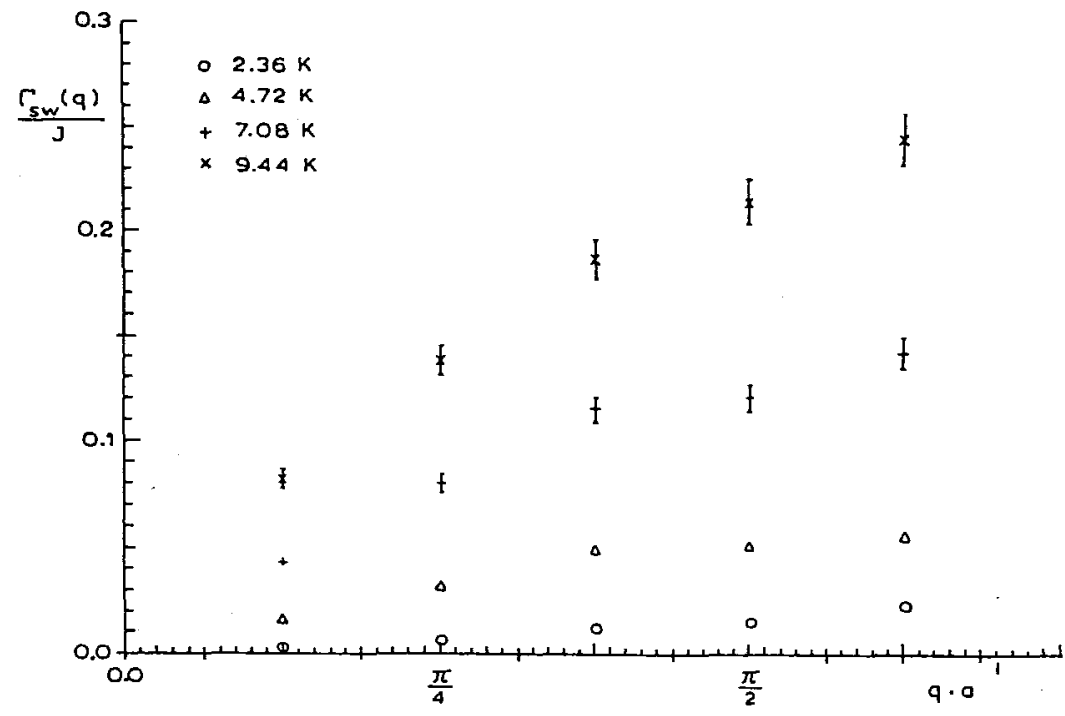

Figure 23: The T-dependence of the spin-wave halfwidths $\Gamma_{s w}$. The polarization is as indicated, the anisotropy $A / k_{B}=9.0 \mathrm{~K}$, and the wave vector $q a=\pi / 8$. The magnetic field $B=1 T(\mathrm{a})$ and $B=2 T(\mathrm{~b})$. The lines show the corresponding sine-Gordon predictions. 


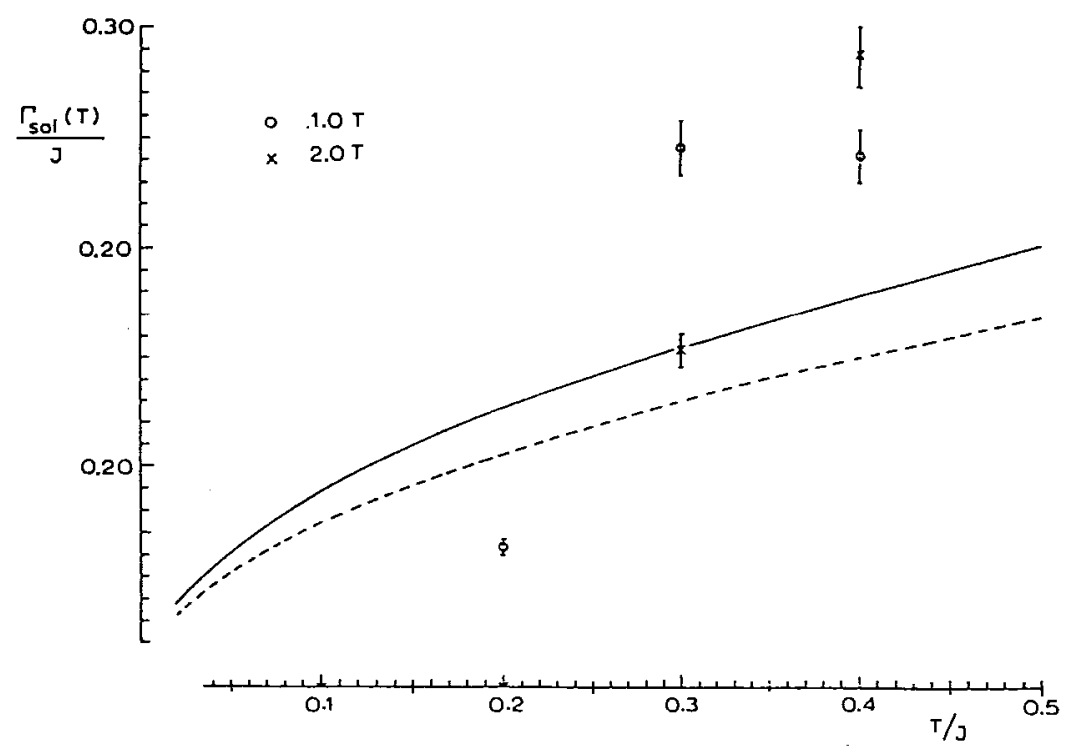

Figure 24: The $T$-dependence of the soliton halfwidths $\Gamma_{\text {sol }}$. The anisotropy $A / k_{B}=9.0 \mathrm{~K}$, and the wave vector $q a=\pi / 8$. The magnetic field is as indicated. The solid and dashed lines show the sine-Gordon predictions for $B=1 T$ and $B=2 T$, respectively.

vectors $q$ the agreement between the sine-Gordon theory, the computer simulations and the experiments is quite good. However, the dispersion is thermally renormalised. For $T=4.7 \mathrm{~K}$ this renormalisation is of the order of $8-10 \%$. This effect has usually been neglected (Kakurai et al., 1990; Steiner et al, 1977).

In view of the interesting narrowing of zero-field in-plane spin-wave line widths observed experimentally (Kakurai et al., 1990) at $4.7 \mathrm{~K}$, we present similar results for $B=1 T$ in Fig. 22. We find that in the presence of the magnetic field the narrowing cannot be observed as clearly as in experiments. The corresponding $\mathrm{T}$-dependence of the $y$ - and $\mathrm{z}$-polarization linewidths is displayed in Fig. 23. Finally, in Fig. 24 we show the T-dependence of the widths of the central peaks for the $y$-polarization.

The half widths of this soliton peak reveal serious disagreement with the sine-Gordon predictions depicted by the solid and dashed lines for 1 and $2 T$, respectively. Our data points show qualitatively different behaviour with respect to the sine-Gordon results. We attribute these deviations to the strong out-ofplane fluctuations. However, in low temperatures the sine-Gordon theory is a reasonable starting point for the analysis of experimental results. For higher temperatures the out-of-plane fluctuations must be included in the theory. 


\section{Conclusions}

In the area of phase transitions the precision of our simulation results reached the sixth decimal place. It was possible due to carefull selection of simulation algorithms, good statistics of the Monte Carlo data and the use of an perturbative transfer matrix approach, yielding directly the magnetization moments. Important was also the combination of different geometries, the asymptotic finite-size scaling analysis and implementation of conformal invariance.

High precision was also reached in the field of quantum spin chains down to low temperatures. Here the combination of of the QTM methods together with the direct finite-size diagonalization technique has been used. Large systems (e.g. $200 \times 14$ for $5=1$ ) were simulated and carefull numerical analysis was applied.

Some unresolved issues in the universal classification of phase transitions in the framework of conformal field theory (Klümper and Pearce, 1993) and new challenging experiments on mesoscopic magnetic particles (Gatteschi, Caneschi, Pardi and Sessoli, 1994) considerably justify further effort in simulation of the low-dimensional magnets.

The spin dynamics study of the classical Heisenberg ferromagnet is only touched here in relation to the easy-plane chains modelling $\mathrm{CsNiF}_{3}$. However, the method is suitable for the dynamic study in higher dimensions (Landau, 1994) and in other systems.

\section{Acknowledgements}

The author is grateful to H. Blöte, L.S. Campana, A. Caramico D'Auria, R. Dekeyser, F. Esposito, U. Esposito, F. Mallezie, G. Musial and P. Pawlicki for their contribution to various parts of the research reviewed here.

The support from the Committee for Scientific Research viagrant No. 8 T11F 01509 is also acknowledged. A part of numerical calculations was performed in the Supercomputing and Networking Center in Poznań.

\section{References}

Achiwa, N. (1969) Linear antiferromagnetic chains in hexagonal ABCl3-type compounds (A; Cs or $\mathrm{Rb}, \mathrm{B} ; \mathrm{Cu}, \mathrm{Ni}$, Co or $\mathrm{Fe}$ ). J. Phys. Soc. Jpn., 27, 561

Aharony, A. and M.E. Fisher (1983) Nonlinear scaling fields and corrections to scaling near criticality. Phys. Rev., B27, 4394

Allroth, E. and H.J. Mikeska (1981) Solitons and magnons in sine-Gordon like magnets. Z. Phys., B43, 209 
Baillie, C.F., R. Gupta, K.A. Hawick and G.S. Pawley (1992) Monte Carlo renormalization group studies of the three-dimensional Ising model. Phys. Rev., B45, 10438

Barber, M.N. (1983) Finite-Size Scaling. In C. Domb and J.L. Lebowitz (Eds.), Phase Transitions and Critical Phenomena, Vol.8, Academic Press, London

Baxter, R.J. (1982) Exactly Solvable Models in Statistical Mechanics. Academic Press, New York

Baxter^ R.J., I.G. Enting and S.K. Tsang (1980) Hard-square lattice gas. J. Stat. Phys., 22, 465

Binder, K. (1981) Finite size scaling analysis of Ising model block distribution functions. Z. Phys., B43, 119

Blöte, H. (1975) The specific heat of magnetic linear chains. Physica, B79, 427

Blöte, H., A. Compa.gner and A. Hoogland (1987) The simple quadratic Ising model with crossing bonds. Physica, A141, 375

Blöte, H. and G. Kamieniarz (1993) Finite-size calculations of the three-dimensional Ising model. Physica, A196, 455

Blöte, H. and G. Kamieniarz (1994) Simulations of the 3D Ising model at criticality. Acta Phys. Polon., A85, 389

Blöte, H. and X.N. Wu (1990) Accurate determination of the critical line of the square Ising antiferromagnet in afield. J. Phys. A: Math. Gen., 23, L627

Bonner, J.C. and M.E. Fisher (1964) Linear magnetic chains with anisotropic coupling. Phys. Rev., A 135, 640

Bruce, A.D. (1985) Universality in the two-dimensional continuous spin model. $J$. Phys. A: Math. Gen., 18, L873

Burkhardt, T.W. and B. Derrida (1985) Moments of the total magnetization and conformal invariance in the finite two-dimensional Ising model. Phys. Rev., B32, 7273

Cachine, J., J.R. Drugovich de Feli and N. Caticha (1989) Non-universal exponents and marginal operators via a Monte Carlo renormalisation-group. J. Phys. A: Math. Gen.,22, 1639

R. Dekeyser, Phys. Rev. B42, 10765 (1990)

Campana, L.S., A. Caramico D'Auria, U. Esposito, G. Kamieniarz and R. Dekeyser (1990) Specific heat of $\left(\mathrm{C}_{6} \mathrm{HnNh}_{3}\right) \mathrm{CuBr}_{3}$ and $\left(\mathrm{C}_{3} \mathrm{HiiNH}_{3}\right) \mathrm{CuCl}_{3}$ within series analysis of finite chain data. Phys. Rev., B42, 10765

Campana, L.S., A. Caramico D'Auria, F. Esposito, U. Esposito, R.W. Gerling, and G. Kamieniarz (1992a) Static prperties of quantum spin $S=1$ chains: TMNB, TMNC and $\mathrm{CsNiF}_{3}$. J. Phys.: Cond. Matter, 4, 2915

Campana L.S., A. Caramico D'Auria, F. Esposito, U. Esposito, G. Kamieniarz (1992b) Finite-Chain approach to the study of CsNiCh- Phys. Rev., B45, 5035 
Campana, L.S., A. Caramico D'Auria, F. Esposito, U. Esposito and G. Kamieniarz (1996), Phys. Rev. B

Cardy, J.L. (1987) Conformal Invariance. In C. Domb and J.L. Lebowitz (Eds.) Phase Transitions and Critical Phenomena Vol. 11 Academic Press, New York

Cuccoli, A., V. Tognetti, P. Verrucchi and R. Vaia (1992) Quantum thermodynamics of the easy-plane ferromagnetic chain. Phys. Rev., B46, 11601

Cullen, J.J. and D.P. Landau (1983) Monte Carlo studies of one-dimensional quantum Heisenberg and XY-models. Phys. Rev., B27, 297

Darriet, J. and L.P. Regnault (1993) The compound $\mathrm{Y}_{2} \mathrm{BaNi}_{5}$ : a new example of a Haldane-gap in $\mathrm{S}=1$ magnetic chain. Solid State Commun., 86, 409

Dekeyser, R. and G. Kamieniarz (1992) Effective field methods with correlations in magnetic systems. J. Magn. Magn. Mat., 104-107, 273

Delica, T. and H. Leschke (1990) Formulation and numerical results of the transfer-matrix method for quantum spin chains. Physica, A168, 736

Delica, T., W.J.M. de Jonge, K. Kopinga, H. Leschke and H.J. Mikeska (1991) Static properties of $\mathrm{CsNiF}_{3}$ : Comparison of numerical results, experimental data and predictions from soliton-bearing models. Phys. Rev., B44, 11773

Dupas, C. and J.P. Renard (1977) Low-temperature magnetic properties of three linear $\mathrm{S}=1$ ferromagnets with an easy plane anisotropy: TMNB, TMNC and $\mathrm{CsNiF}_{3}$. J. Phys. C: Solid State Phys., 10, 5057

Ferrenberg, A.M. and D.P. Landau (1991) Critical behavior of the three-dimensional Ising model: a high resolution Monte Carlo study. Phys. Rev., B44, 5081

Fisher, M.E. and H. Au-Yang (1979) Inhomogeneous differential approximants for power series. J. Phys. A: Math. Gen., 12, 1677

Gadet, V., M. Verdaguer, V. Briois, A. Gleizes, J.P. Renard, P. Beauvillain, C. Chappert, T. Goto, K. Le Dang and P. Veillet (1991) Structural and magnetic properties of $\left(\mathrm{CH}_{3}\right)_{4} \mathrm{NNi}\left(\mathrm{NO}_{2}\right)_{3}$ : a Haldane-gap system. Phys. Rev., B44, 705

Gatteschi, D., A. Caneschi, L, Pardi and R. Sessoli (1994) Large clusters of metal ions: the transition from molecular to bulk magnets. Science, 265, 1054

Gerling, R.W. and D.P. Landau (1990) Spin dynamics study of the classical ferromagnetic XY chain. Phys. Rev., B41, 7139

Grille, H., G. Kamieniarz and R.W. Gerling (1992) Spin dynamics of the classical easy-plane Heisenberg chain. J. Magn. Magn. Mat., 104-107, 1067

Halperin, B.I. (1992) Quantum antiferromagnets in one and two dimensions. J. Magn. Magn. Mat., 104-107, 761

Indekeu, J.O., A.Maritan and A.L.Stella (1982) Renormalization group recursions by mean-field approximations. J. Phys. A: Math. Gen. 15, L291

de Jongh, L.J. and A.R. Miedema (1974) Experiments on simple magnetic model systems. Adv. Phys., 23, 1 
Kakurai, K., M. Steiner and B. Dorner (1990) Study of the spin weave linewidth in ID easy-plane ferromagnet using neutron polarization analysis. Europhys. Lett., 12,653

Kamieniarz, G., F. Mallezie and R. Dekeyser (1988) Magnetization and correlation length of the easy-plane ferromagnetic chain: numerical analysis and application to $\left(\mathrm{C}_{6} \mathrm{HilNH}_{3}\right) \mathrm{CuBr} 3$. Phys. Rev., B38, 6941

Kamieniarz, G. and H.W.J. Blöte (1993a) Universal ratio of magnetization moments in two-dimensional Ising model. J. Phys. A: Math. Gen., 26, 201

Kamieniarz, G. and H. Blöte (1993b) The non-interacting hard-square lattice gas: Ising universality. J. Phys. A: Math. Gen., 26, 6679

Kamieniarz, G., G. Musial and R. Dekeyser (1994) Effective field methods for magnetic systems. Acta Phys. Polon., A85, 413

Kamieniarz, G. and R. Matysiak (1996a), in preparation

Kamieniarz, G., G. Musial and R. Dekeyser (1996b), in preparation

Klümper, A. and P.A. Pearce (1993) New results for exactly solvable critical RSOS models and vertex models in two dimensions. Physica, A194, 397

Kopinga, K., J. Emmen, G.C. de Vries, L.F. Lemmens and G. Kamieniarz (1988) Comparison between experiment and numerical calculations on $\mathrm{S}=1 / 2$ HeisenbergXY ferromagnetics chains. J. de Physique, C8, 1451

Kopinga, K., T. Delica and H. Leschke (1989) Static properties of an easy-plane ferromagnetics $\mathrm{S}=1 / 2$ chain: comparison of numerical results and experimental data on $\left(\mathrm{C}_{6} \mathrm{HiiNH}_{3}\right) \mathrm{CuBr}_{3}$. Phys. Rev., B40, 7239

Kopinga, K., T. Delica, H. Leschke and I. Riedel (1993) Static properties of a ferromagnetic $S=1 / 2$ chain system with orthorombic exchange anisotropy: comparison of numerical results and experimental data on $\left.(\mathrm{CgITu} \mathrm{NH})_{3}\right) \mathrm{CuCl}_{3}$. Phys. Rev., B47, 5447

Landau, D.P. (1994) Computer simulation studies of critical phenomena. Physica, A 205, 41

Le Guillou, J.C. and J. Zinn-Justin (1980) Critical exponents from field theory. Phys. Rev., B21, 3976

Liu, A.J. and M.E. Fisher (1989) The three-dimensional Ising model revisited numerically. Physica, A156, 35

Livet, F. (1991) The cluster updating Monte Carlo algorithm applied to the 3D Ising problem. Europhys. Lett., 16, 139

Manousakis, E. (1991) The spin- $\frac{1}{2}$ Heisenberg antiferromagnet on a square lattice and its applications to the cuprous oxides. Rev. Mod. Phys., 63, 1

Mikeska, H.J. (1978) Solitons in a one-dimensional magnet with an easy plane. $J$. Phys. G: Solid State Phys., 11, L29 
Mikeska, H.J. and M. Steiner (1991) Solitary excitations in one-dimensional magnets. Adv. Phys., 40, 191

Morra, R.M., R.M. Armstrong, W.J.L. Buyers and H. Hirakawa (1988) Spin dynamics and the Haldane gap in the spin-1 quasi-one-dimensional antiferromagnet $\mathrm{CsNiCl}_{3}$. Phys. Rev., B38, 543

Moses, D., E. Ehrenfreund, J. Makovsky and H. Shechter (1977) Magnetic specific heat of the nearly one-dimensional antiferromagnet $\mathrm{CsNiCl}_{3}$. J. Phys. C: Solid State Phys., 10, 433

de Neef, T. (1976) Thermodynamics of magnetic chains with $\mathrm{S} \leq 5 / 2$. Phys. Rev, B13, 4141

Niemeijer, Th. and J.M.J, van Leeuwen (1976) Renormalization theory for Isinglike spin systems. In C. Domb and M.S. Green (Eds.) Phase Transitions and Critical Phenomena, Vol. 6, Acad. Press, London, New York and San Francisco, p. 425

Nickel, B.G. and J.J. Rehr (1990) High-Temperature Series for Scalar Field Lattice Models: Generations and Analysis J. Stat. Phys., 61, 1

Pawlicki, P., G. Kamieniarz and J.Rogiers (1995) Phase diagram of the extended Ashkin-Teller model. J. Magn. Magn. Mat., 140-144, 1471

Pearce, P.A. and K.A. Seaton (1990) The off-critical integrable Ashkin-Teller model. J. Phys. A: Math. Gen., 23, 1191

Privman, V. and M.E. Fisher (1984) Universal critical amplitudes in finite-size scaling. Phys. Rev., B30, 322

Racz, Z. (1980) Phase boundary of Ising antiferromagnets near $H=H_{c}$ and $T=0$ : Results from hard-core lattice gas calculations. Phys. Rev., B21, 4012

Ramirez, A.P. and W.P. Wolf (1985) Specific heat of CsNiF 3 . Phys. Rev., B32, 1639

Ramirez, A.P., S.-W. Cheong and M.L. Kaplan (1994) Specific heat of defects in Haldane systems $\mathrm{Y}_{2} \mathrm{BaNiO}_{5}$ and NENP: absence of the spin-1/2 excitations. Phys.Rev. Lett, 72, 3108

Rosengren, A. (1986) On the combinatorial solution of the Ising model. J. Phys. A: Math. Gen., 19, 1709

Steiner, M., J. Villain and C.G. Windsor (1976) Theoretical and experimental studies on one-dimensional magnetic systems. Adv. Phys., 25, 1

Steiner, M. and J.K. Kjems (1977) Spin waves in $\mathrm{CsNiF}_{3}$ with an applied magnetic field. J. Phys. C: Solid State Phys., 10, 2665

Steiner, M., K. Kakurai and J.K. Kjems (1983) Experimental Study of the Spindynamics in the 1-D Ferromagnet with Planar Anisotropy: $\mathrm{CsNiF}_{3}$ in an External Magnetic Field. Z. Phys., B53, 117 
Suzuki M. (1993) Quantum Monte Carlo methods - recent developments. Physica, A194, 432

Swendsen, R.H. and J.-S. Wang (1988) Nonuniversal Critical Dynamics in Monte Carlo Simulations. Phys. Rev. Lett., 58, 86

Weng, C.J. (1968) Ph. D. Thesis, Cornegie Institute of Technology

Wolff, U. (1989) Collective Monte Carlo Updating for Spin System. Phys. Rev. Lett, 62, 361

Wood, D.W. and M. Goldfinch (1980) Vertex models for the hard-square and hard hexagon gases, and critical parameters from the scaling transformations. J. Phys. A: Math. Gen., 13, 2781 